\title{
Oceanographic conditions west of the Baja California coast, 2002-2003: A weak El Niño and subarctic water enhancement
}

\section{Condiciones oceanográficas frente a la costa occidental de Baja California, 2002-2003: Influencia de un El Niño débil y del incremento de agua subártica}

\author{
Reginaldo Durazo ${ }^{1 *}$ \\ Gilberto Gaxiola-Castro² \\ Bertha Lavaniegos ${ }^{2}$ \\ Rubén Castro-Valdez ${ }^{1}$ \\ José Gómez-Valdés ${ }^{2}$ \\ Affonso Da S. Mascarenhas Jr. ${ }^{3}$ \\ ${ }^{1}$ Facultad de Ciencias Marinas \\ ${ }^{3}$ Instituto de Investigaciones Oceanológicas \\ Universidad Autónoma de Baja California \\ Apartado postal 453 \\ Ensenada, CP 22860, Baja California, México \\ *E-mail: rdurazo@uabc.mx \\ ${ }^{2}$ Centro de Investigación Científica y de Educación Superior de Ensenada \\ Km. 107 carretera Tijuana-Ensenada \\ Ensenada, Baja California, México
}

Recibido en abril de 2004; aceptado en abril de 2005

\begin{abstract}
Oceanographic conditions in the Pacific waters off Baja California are described for the period 2002-2003. Anomalies in the temperature-salinity relationships were observed from October 2002 to April 2003, with a clear contribution of low salinity and low temperature subarctic water in the upper $100 \mathrm{~m}$ of the water column. Below $100 \mathrm{~m}$, physical data suggested weak El Niño conditions. Weakened or poleward winds were not observed. The pelagic ecosystem during the intrusion of subarctic waters showed strong seasonal changes, with localized centers of high chlorophyll and zooplankton in July 2002, followed by a strong decrease in the following autumn and winter seasons. Both chlorophyll and zooplankton rebounded in April 2003, but zooplankton showed a stronger onshore-offshore gradient. There was no strong biological signal. The zooplankton taxa that showed an increase during El Niño 2002-2003 were salps and chaetognaths. The increase of salps did not appear to be related to this event, due to their seasonal occurrence limited to the northern region. In contrast the increase of chaetognaths presented a similar pattern to that observed in El Niño 1997-1998.
\end{abstract}

Key words: El Niño, California Current System, Baja California, bio-physical coupling.

\section{Resumen}

En este trabajo se describen las condiciones oceanográficas del Océano Pacífico frente a Baja California durante el periodo 2002-2003. De octubre de 2002 a abril de 2003 se observaron relaciones anómalas entre la temperatura y la salinidad debidas a la fuerte presencia de agua de origen subártico, con baja salinidad y baja temperatura, en los 100 m superiores de la columna de agua. Por debajo de los $100 \mathrm{~m}$ de profundidad los datos físicos sugirieron condiciones que indican la presencia de un evento El Niño débil. Durante el periodo de estudio no se observó una disminución en los vientos dominantes o vientos con dirección hacia el polo. El ecosistema pelágico mostró fuertes cambios estacionales debidos a la intrusión de agua subártica, con zonas de alta clorofila y zooplancton en julio de 2002, con un fuerte decremento durante el otoño e invierno siguientes. Tanto la clorofila como el zooplancton resurgieron en abril de 2003, con un mayor gradiente costa-océano en el zooplancton. No se presentó una fuerte señal biológica durante el período. Los grupos de zooplancton que mostraron un incremento durante El Niño 2002-2003 fueron las salpas y los quetognatos. El incremento de salpas no parece estar asociado al evento, ya que su incidencia fue estacional y restringida a la región norte. En cambio el incremento en quetognatos presentó un patrón similar al de El Niño 1997-1998.

Palabras clave: El Niño, Sistema de la Corriente de California, Baja California, acoplamiento físico-biológico. 


\section{Introduction}

The California Current System (CCS) is comprised of the near-surface equatorward California Current (CC), the subsurface poleward California Undercurrent (CU), and the nearshore seasonally intermittent California Countercurrent (CCC). These currents change in strength at seasonal and interannual scales. Interannual variability is closely linked to changes in the mid-latitude atmospheric circulation associated with El Niño events. During El Niño, the Aleutian low pressure cell strengthens and produces stronger winter storms (Strub and James, 2003), weakened clockwise (cyclonic anomaly) atmospheric circulation (Schwing et al., 2002), increased onshore Ekman transport and a deeper thermocline. These were in general the changes observed along the northeastern Pacific during the 1997-1998 El Niño event.

The anomalous conditions off Baja California described above coincided with near-surface coastal poleward currents, warmer and saltier than usual waters (up to $9^{\circ} \mathrm{C}$ and 0.8 , respectively), an expansion of the volume of the CU (Durazo and Baumgartner, 2002), and a shifting in the species composition of zooplankton (Lavaniegos et al., 2002). The conditions observed were seen as the response to anomalous cyclonic atmospheric circulation, which displaced the CC core offshore and favored the poleward intrusion of subtropical waters into the coastal region (Durazo and Baumgartner, 2002).

During the period 2002-2003, an El Niño event took place. For the equatorial Pacific, this climatic episode was described as moderate (McPhaden, 2004), with sea surface temperature (SST) anomalies developing between September and December 2002. These SST anomalies occurred in conjunction with strong, sustained eastward surface current anomalies that began in July (Lagerloef et al., 2003) and were detected in the eastern equatorial Pacific in the second half of 2002 (McPhaden, 2004). Within the CCS, El Niño 2002-2003 was marked by cold and fresh surface waters during 2002 and weak warming in early 2003 (Venrick et al., 2003). Off California, cold and fresh conditions have been associated with an excess of subarctic water (Bograd and Lynn, 2003). There have been no reports that indicate such conditions off Baja California. The purpose of this paper is to analyze the oceanographic conditions in the southern portion of the CCS during El Niño 2002-2003, using hydrographic data collected during this period, and define whether subarctic waters reached southern latitudes. Relevant biological data were also analyzed to study the pelagic ecosystem response.

\section{Methods}

Winds were measured using Aanderaa meteorological stations at four selected locations along the northern Baja California coast, from January 2000 to July 2003. These include two coastal stations, one at Isla Todos Santos (ITS) and another at Bahía San Quintín (BSQ); one inland station at San Quintín (SQ); and an oceanic station at Isla Guadalupe (IG) (see table 1 and fig. 1). The ITS station is located in the

\section{Introducción}

El Sistema de la Corriente de California (SCC) está constituido por una corriente superficial con dirección al ecuador llamada Corriente de California (CC), por la Subcorriente de California (CU) que tiene un flujo subsuperficial con dirección hacia el polo y por la Contracorriente de California (CCC) que es costera y con una estacionalidad intermitente. Estas corrientes cambian en intensidad a escalas estacionales e interanuales. La variabilidad interanual está fuertemente relacionada con los cambios en la circulación atmosférica de latitudes medias, asociada a eventos El Niño. Durante un El Niño la celda de baja presión de las Aleutianas se fortalece y produce tormentas invernales muy severas (Strub y James, 2003), debilitamiento de la circulación anticiclónica (anomalía ciclónica) de la atmósfera (Schwing et al., 2002), incremento del transporte de Ekman hacia la costa y hundimiento de la termoclina. En general, éstos fueron los cambios observados a todo lo largo del Pacífico nororiental durante El Niño 1997-1998.

Frente a Baja California, las condiciones anómalas descritas anteriormente coincidieron con corrientes costeras superficiales hacia el polo, agua más caliente y salada que lo normal (aumentos de $9^{\circ} \mathrm{C}$ y 0.8 , respectivamente), una expansión del volumen de la CU (Durazo y Baumgartner, 2002) y un cambio en las especies que componen la comunidad del zooplancton (Lavaniegos et al., 2002). Las variaciones en las condiciones oceanográficas se observaron como respuesta a la anomalía en la circulación ciclónica de la atmósfera, la cual desplazó el núcleo de la CC hacia fuera de la costa y favoreció la intrusión de agua subtropical en la región costera de Baja California con dirección hacia del polo (Durazo y Baumgartner, 2002).

Durante el periodo 2002-2003 se presentó un evento El Niño en el Océano Pacífico. Este episodio climático fue descrito como moderado para el Pacífico Ecuatorial (McPhaden, 2004), con anomalías en la temperatura superficial del mar (TSM) desarrolladas entre septiembre y diciembre de 2002. Estas anomalías en TSM ocurrieron junto con anomalías sostenidas en el flujo de la corriente superficial con dirección al este que iniciaron en julio (Lagerloef et al., 2003), las cuales fueron detectadas en el este del Océano Pacífico Ecuatorial durante el segundo semestre de 2002 (McPhaden, 2004). Dentro del SCC la influencia de El Niño 2002-2003 fue identificada por la presencia de agua fría y menos salada cercana a la superficie durante 2002 y un débil calentamiento a inicios de 2003 (Venrick et al., 2003). Frente a California el escenario de agua fría y menos salada ha sido asociado con el exceso de agua proveniente del subártico (Bograd y Lynn, 2003). No existen trabajos que indiquen que esta situación se extendió hacia el sur frente a Baja California. El propósito de esta contribución es el de analizar las condiciones oceanográficas en la porción sur del SCC durante El Niño 2002-2003 a partir de datos hidrográficos recolectados durante ese periodo y definir si el agua del subártico alcanzó estas latitudes sureñas. Se analizó además información biológica relevante para estudiar la respuesta del ecosistema pelágico. 
northern part of the island, about $15 \mathrm{~km}$ west of Ensenada. The BSQ station is located near the shoreline north of San Quintín Bay, while the SQ station is about $20 \mathrm{~km}$ inland. The IG station is located in the southern part of the island. The time frame of measurements differs among the four stations but the basic statistics of all data at each station are included here in order to provide a reference for future works.

Starting in November 1998, SST was measured at IG adjacent to the meteorological station, using a SBE 26 SEAGAUGE. Accuracy and resolution of the temperature sensor are $0.02^{\circ} \mathrm{C}$ and $0.01^{\circ} \mathrm{C}$, respectively. An analysis of the temperature time series from 2000 to 2003 is presented, although a seasonal mean was obtained with all available data.

Since 1997, the Investigaciones Mexicanas de la Corriente de California (IMECOCAL) program conducts quarterly surveys off Baja California along a subset grid of the original California Cooperative Fisheries Investigations (CalCOFI) program (fig. 1). This report analyzes data gathered mainly during cruises conducted in July and October 2002, and January/February and April 2003 (hereafter cruises 0207, 0210, 0301 and 0304, respectively). Some previous data are also analyzed. At each oceanographic station, CTD casts were made to $1000 \mathrm{~m}$ (bottom depth permitting) and water samples were collected from seven depths $(0,10,20,50,100,150$ and $200 \mathrm{~m}$ ) for the determination of dissolved oxygen and chlorophyll $a$. To determine chlorophyll $a$, samples were screened through Whatman GF/F filters with $0.7-\mu \mathrm{m}$ nominal pore size in order to collect phytoplankton cells, and analyzed by the fluorometric technique with a Turner Designs AU-05 fluorometer (Holm Hansen et al., 1965; Yentsch and Menzel, 1963; Venrick and Hayward, 1984).

Standard (0.505-mm mesh) double oblique bongo tows from $210 \mathrm{~m}$ depth (or from $10 \mathrm{~m}$ above the bottom) to the surface were carried out at each station. A flow meter in the net entrance was used to measure the volume of water strained (Smith and Richardson, 1977). Zooplankton volume was measured by displacement in a graduated cylinder (Smith and Richardson, 1977), one month after the end of each cruise. Taxonomic analyses were done for cruises 0207 and 0301, and for previous winter and summer IMECOCAL cruises (9801, 9807, 9901, 9908 and 0001). Samples were subdivided with a Folsom splitter to $1 / 8$ or $1 / 16$ and in some cases to $1 / 32$. All specimens in the subsample were counted. Zooplankton volume and abundance were standardized to $1 \mathrm{~m}^{3}$ of filtered water. Mean and confidence intervals were estimated from logtransformed data. Zooplankton volume data from previous CalCOFI cruises conducted off Baja California were used to estimate seasonal anomalies. First, CalCOFI data collected in January, April, July and October from 1951 to 1984 were selected and log-transformed. Then, seasonal means for the period 1951-1984 were computed. Finally, mean zooplankton volumes for each cruise (0207, 0210, 0301 and 0304) were compared to seasonal means to compute anomalies. The area was divided into north (lines 100-110) and central (lines 113130) regions as described in Lavaniegos et al. (2002).

\section{Métodos}

El viento fue medido entre enero de 2000 y julio de 2003 con un estación meteorológica Aanderaa en cuatro puntos seleccionados a lo largo de la costa norte de Baja California. Las mediciones se hicieron en dos estaciones costeras de Isla Todos Santos (ITS) y Bahía San Quintín (BSQ), en una estación alejada de la costa en San Quintín (SQ) y en una estación oceánica instalada en Isla Guadalupe (IG) (ver tabla 1 y fig. 1). La estación de ITS se localizó al norte de la isla, aproximadamente $15 \mathrm{~km}$ al oeste de la ciudad de Ensenada. La estación de BSQ está cerca de la costa al norte de Bahía de San Quintín, mientras que la estación SQ está aproximadamente 20 $\mathrm{km}$ tierra adentro. La estación IG se localiza al sur de Isla Guadalupe. Los tiempos de muestreo difieren entre las cuatro

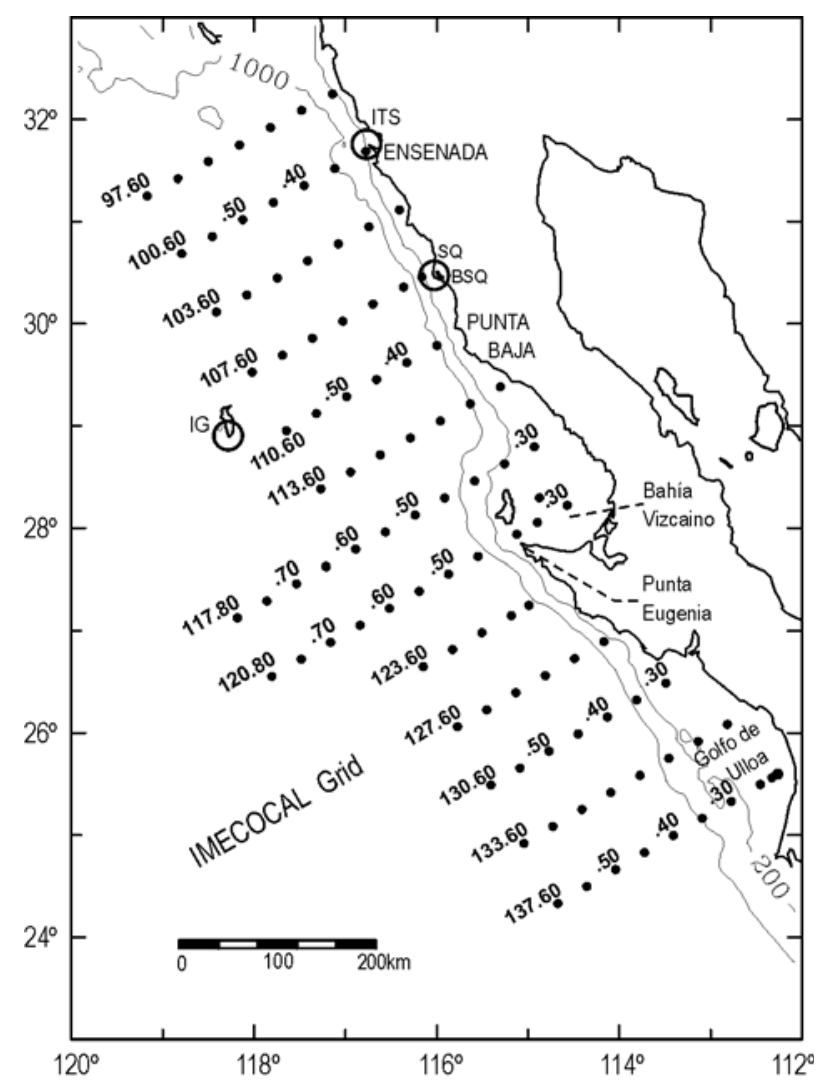

Figure 1. The IMECOCAL sampling grid showing the regular station pattern between lines 97 and 137. Line 97 was sampled only during the spring cruise. (•) Stations. (O) Locations from where wind and sea surface temperature data were collected. ITS = Isla Todos Santos, IG = Isla Guadalupe, SQ = San Quintín and BSQ = Bahía San Quintín. The 200- and 1000-m depth contours are included.

Figura 1. Malla del muestreo IMECOCAL donde se muestra el patrón regular de las estaciones muestreadas entre las líneas 97 y 137. La línea 97 fue muestreada solamente durante el crucero de primavera. (•) Estaciones. (O) Sitios de recolección de datos de viento y TSM. ITS = Isla Todos Santos, IG = Isla Guadalupe, SQ = San Quintín y BSQ = Bahía San Quintín. Se incluyen los contornos que marcan los 200 y 1000 m de profundidad. 


\section{Results}

\section{Wind observations}

For each station, ellipse components were computed in order to determine the axis of maximum variability. Because the coastline and major axis orientations are very similar (table 1), wind velocity components were rotated so as to define alongshore and across-shore components to coincide with the major and minor axes, respectively. Time series of the alongshore wind component for all stations, as well as SST at IG superimposed on the annual fit, are shown in figure 2. Right panels (fig. 2a-d) denote wind direction frequency distributions. In this paper, wind and SST anomalies are defined as those observations above (weaker wind or lower SST) or below (stronger wind or higher SST) the seasonal mean of the alongshore component (or SST), plus or minus the standard error (shaded band in fig. 2). It is important to note that since records are short, seasonal fits may not adequately reflect the true seasonal variability. The results may therefore change at a later stage when larger data sets are obtained.

Data reveal generally equatorward winds (negative alongshore component) and a dominance of northwesterly $\left(\sim 315^{\circ}\right)$ winds, strongest around May/June and weakest in January. Except for the SQ station (inland, fig. 2c), a relative maximum in intensity is also seen in October. At the ITS and SQ coastal stations (fig. 2a, c), large variability in the frequency distributions and the presence of winds in the cross-shore direction suggest the influence of the local topography and breezes. The amplitude of the seasonal mean is larger for the oceanic station $\left(\sim 2 \mathrm{~m}^{-1}\right.$ at IG, fig. $\left.2 \mathrm{~d}\right)$. The IG station presents the more intense and persistent winds, with high variability of alongshore wind speed around the seasonal mean. The ITS station shows the largest variability relative to SQ and BSQ, due to the diurnal breeze. Mean wind speed (table 1) is maximum at the oceanic station (IG) and minimum at the inland location (SQ). Anomalously weak winds signaling El Niño conditions were not recorded at any of the stations, except for events lasting two to three weeks at IG during winter (late 2002 and early 2003).

On the other hand, the SST time series for IG exhibits a strong seasonal cycle, with a relative maximum $\left(\sim 20^{\circ} \mathrm{C}\right)$ around September and a minimum $\left(\sim 15^{\circ} \mathrm{C}\right)$ around March (fig. 2e). Above normal temperatures were observed from January to September 2000 and in early 2003.

\section{Thermohaline characteristics}

Temperature-salinity (T-S) diagrams are used here to illustrate the main thermohaline characteristics that prevailed during the surveys (fig. 3). In order to better visualize changes in the existing conditions, T-S data pairs are contrasted to climatological profiles ( \pm 1 standard deviation) for each season, computed from the 1950-1978 CalCOFI and the 1997-2003 IMECOCAL data. Mean temperature and salinity were computed over selected density ranges. Red and green thick curves estaciones. Sin embargo, en este trabajo se incluye la estadística básica de todos los datos de cada estación, con el fin de proveer una referencia para futuras contribuciones en el área de estudio.

A partir de noviembre de 1998 se midió la TSM en IG con un sensor SBE 26 SEAGAUGE, en un lugar adyacente a la estación meteorológica. La precisión y la resolución del sensor de temperatura son $0.02^{\circ} \mathrm{C}$ y $0.01^{\circ} \mathrm{C}$, respectivamente. En este trabajo se presenta un análisis de las series de tiempo de la temperatura medida entre los años 2000 y 2003, referidos a una media estacional a partir de todos los datos disponibles.

Desde 1997 el programa Investigaciones Mexicanas de la Corriente de California (IMECOCAL) realiza cruceros trimestrales frente a Baja California, a lo largo de una malla reducida de las estaciones originales ocupadas históricamente por el programa California Cooperative Oceanic Fisheries Investigations (CalCOFI) (fig. 1). En este trabajo se analizan principalmente los datos obtenidos durante los cruceros de julio y octubre de 2002, los cruceros de enero/febrero y abril de 2003 (nombrados como 0207, 0210, 0301 y 0304, respectivamente), así como datos previos a estos años. Cada estación oceanográfica incluyó lances de CTD hasta $1000 \mathrm{~m}$ (o hasta donde fue permitido por el fondo) y colecta de muestras de agua a siete profundidades $(0,10,20,50,100,150$ y $200 \mathrm{~m})$ para la determinación de oxígeno disuelto y clorofila $a$. La clorofila fue medida en el fitoplancton recolectado a través de filtros Whatman GF/F con poros de $0.7 \mu \mathrm{m}$. Las muestras recolectadas fueron analizadas por la técnica fluorimétrica con un fluorímetro Turner Designs AU-05 (Holm Hansen et al., 1965; Yentsch y Menzel, 1963; Venrick y Hayward, 1984).

En cada una de las estaciones se hicieron lances oblicuos con una red bongo estándar (malla de $0.505 \mathrm{~mm}$ ) desde los $210 \mathrm{~m}$ de profundidad (o desde $10 \mathrm{~m}$ por arriba del fondo). Para medir el volumen del agua tamizada se instaló un flujómetro en la entrada de la red (Smith y Richardson, 1977). El volumen del zooplancton recolectado fue determinado un mes después del término de cada crucero por el método del líquido desplazado medido con una probeta graduada (Smith y Richardson, 1977). Los análisis taxonómicos fueron hechos para los cruceros 0207, 0301 y para los cruceros IMECOCAL de inviernos y veranos anteriores (9801, 9807, 9901, 9908 y 0001). Las muestras fueron subdivididas con un separador Folsom a 1/8 ó 1/16 y en algunos casos a 1/32. Todos los especímenes presentes en las muestra fueron contados. Tanto el volumen del zooplancton como su abundancia fueron estandarizados a $1 \mathrm{~m}^{3}$ de agua filtrada. La media y los intervalos de confianza fueron estimados de los datos transformados a logaritmos. Para estimar las anomalías estacionales se utilizaron los datos del volumen del zooplancton de cruceros CalCOFI realizados con anterioridad frente a Baja California. Primeramente los datos CalCOFI de enero, abril, julio y octubre recolectados entre 1951 y 1984 fueron seleccionados y transformados a logaritmos. Posteriormente se calcularon las medias estacionales para el periodo 1951-1984. Finalmente, las medias del volumen del zooplancton para cada crucero 
Table 1. Alongshore wind speed statistics for stations located at Isla Todos Santos (ITS), Bahía San Quintín (BSQ), San Quintín (SQ) and Isla Guadalupe (IG). Coastline orientation was obtained from a detailed chart.

Tabla 1. Estadísticas del viento paralelo a la costa medido en la estaciones de Isla Todos Santos (ITS), Bahía San Quintín (BSQ), San Quintín (SQ) e Isla Guadalupe (IG). La orientación de la línea de costa fue obtenida a partir de un mapa.

\begin{tabular}{cccccc}
\hline Station & Position & Period of measurements & $\begin{array}{c}\text { Coast orientation } \\
\text { (true North) }\end{array}$ & $\begin{array}{c}\text { Mean speed } \\
\left(\mathrm{m} \mathrm{s}^{-1}\right)\end{array}$ & $\begin{array}{c}\text { Mean } \\
\text { direction }\end{array}$ \\
\hline ITS & $31.8^{\circ} \mathrm{N}, 116.8^{\circ} \mathrm{W}$ & Feb 2002-Jun 2003 & 328 & $3.43 \pm 1.7$ & $325^{\circ}$ \\
BSQ & $30.5^{\circ} \mathrm{N}, 116.0^{\circ} \mathrm{W}$ & Jun 2002-Jul 2003 & 333 & $3.73 \pm 2.1$ & $326^{\circ}$ \\
SQ & $30.5^{\circ} \mathrm{N}, 115.8^{\circ} \mathrm{W}$ & Jan 2001-Dec 2002 & 333 & $2.90 \pm 1.8$ & $342^{\circ}$ \\
IG & $28.9^{\circ} \mathrm{N}, 118.2^{\circ} \mathrm{W}$ & Apr 2000-May 2003 & n.a. & $6.03 \pm 2.9$ & $319^{\circ}$ \\
\hline
\end{tabular}

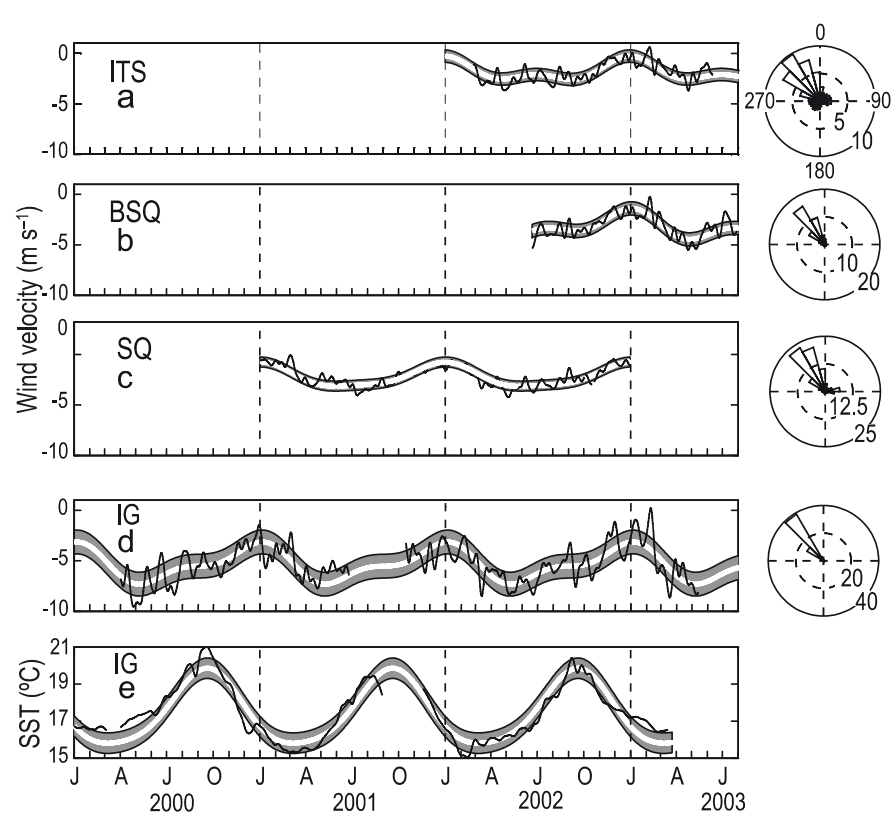

Figure 2. Time series of daily-averaged alongshore (major axis of ellipse component) winds $\left(\mathrm{m} \mathrm{s}^{-1}\right.$ ) for the available records during 2000-2003 at the four stations along Baja California. The bottom panel is the time series of daily-averaged sea surface temperature measured at Isla Guadalupe. Bold white lines represent the biharmonic annual climatological cycle. Shaded areas are the standard errors for each day. Series have been smoothed with a 7-day running mean. Right panels are the wind direction frequency distributions for each location.

Figura 2. Series de tiempo del viento promedio diario paralelo a la costa (eje mayor de la elipse componente; $\mathrm{m} \mathrm{s}^{-1}$ ) para los datos disponibles durante 2000-2003 en cuatro estaciones de Baja California. La figura inferior es la serie de tiempo de los promedios diarios de la TSM medida en Isla Guadalupe. Las líneas blancas gruesas son la climatología del ciclo anual en base a dos armónicos. Las áreas sombreadas representan el error estándar para cada día. Las series han sido suavizadas con una media corrida de 7 días. Las figuras del lado derecho representan la distribución de las frecuencias en la dirección del viento para cada localidad.

depict the climatological and cruise means, respectively. Care was taken to compare T-S diagrams and the corresponding mean profiles for the same oceanic stations occupied during each cruise. Cruise mean curves (green) indicate that near normal conditions persisted during the summer of 2002 (fig. 3a),
(0207, 0210, 0301 y 0304) fueron comparadas con las medias estacionales para calcular las anomalías. El área de estudio fue dividida en regiones norteña (líneas 100-110) y central (líneas 113-130) de acuerdo con el criterio de Lavaniegos et al. (2002).

\section{Resultados}

\section{Observaciones del viento}

Para determinar los ejes de variabilidad máxima del viento se calcularon las elipses componentes en cada una de las estaciones meteorológicas. Debido a que los ejes de la línea de costa y de las orientaciones del eje prinicipal son muy similares (tabla 1), las componentes de la velocidad del viento fueron rotadas para que las componentes paralelas a la costa y perpendiculares a la costa coincidieran con los ejes mayores y menores, respectivamente. Las series de tiempo de la componente del viento paralela a la costa de todas las estaciones, así como la TSM en IG superpuesta a un ajuste anual, se muestran en la figura 2. Las gráficas en el lado derecho (fig. 2a-d) muestran la distribución de la frecuencia de la dirección del viento. En este trabajo las anomalías del viento y de la TSM son definidas como aquellas observaciones por arriba (viento débil o menor TSM) o por debajo (viento fuerte o mayor TSM) de la media estacional de la componente paralela a la costa (o TSM) más menos el error estándar (banda sombreada en la fig. 2). Es necesario notar que debido a que las series de datos son cortas, los ajustes estacionales pueden no reflejar adecuadamente la variabilidad estacional verdadera. Los resultados por lo tanto podrían cambiar en una etapa posterior cuando se obtenga una mayor cantidad de datos.

La información analizada mostró vientos predominantes con dirección al ecuador (componente negativa a lo largo de la costa) y una dominancia de vientos del noroeste $\left(\sim 315^{\circ}\right)$, los cuales fueron más fuertes durante mayo/junio y débiles en enero. Durante octubre se presentó una intensidad relativamente alta del viento, excepto en la estación SQ (fig. 2c). La gran variabilidad en la frecuencia de distribución del viento y la presencia de vientos con dirección perpendicular a la costa presente en las estaciones costeras ITS y BSQ (fig. 2a, c) sugiere la influencia de la topografía y de las brisas locales. La amplitud de la media estacional del viento fue mayor en la 


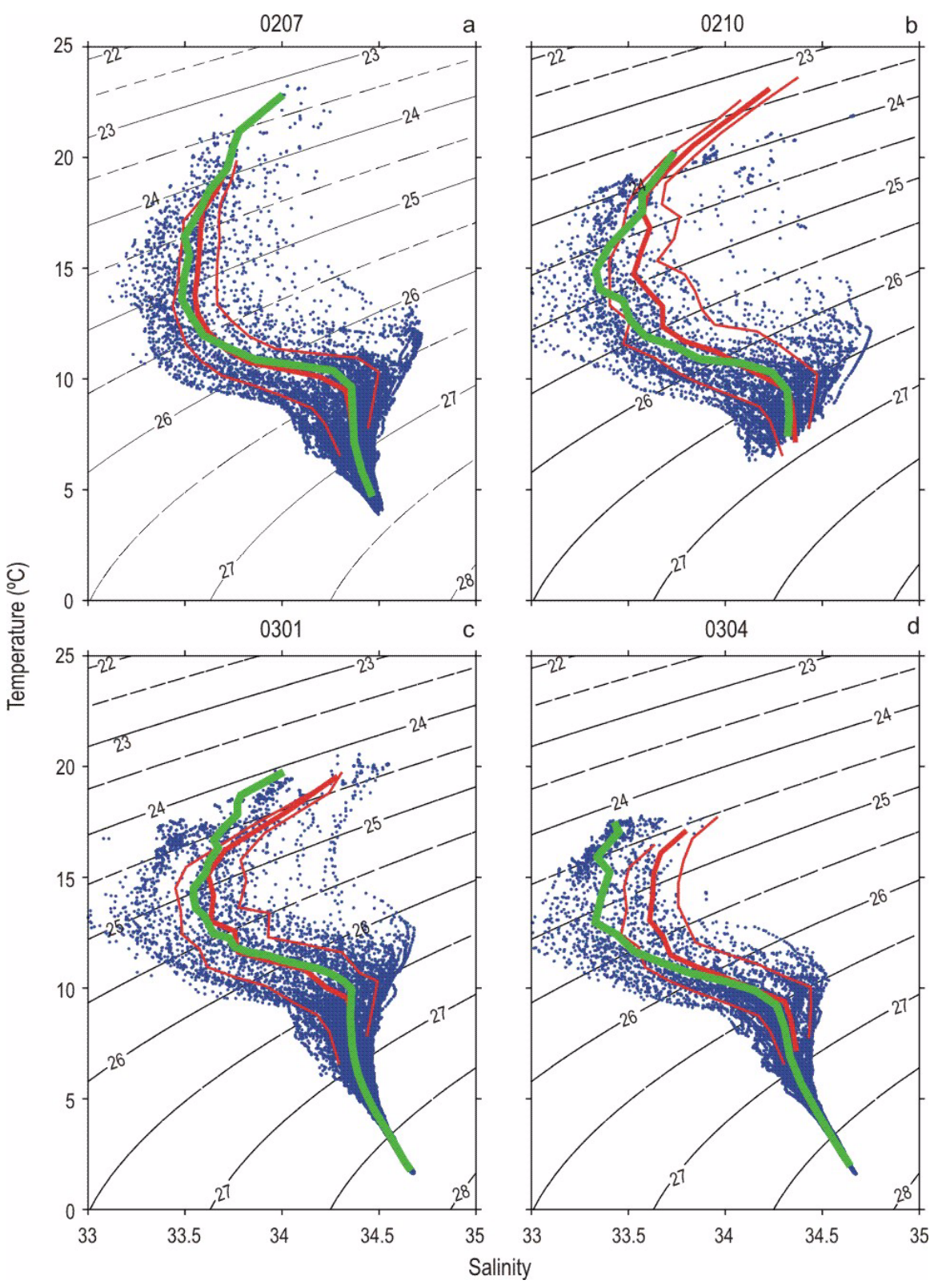

Figure 3. Temperature-salinity (T-S) diagrams from the IMECOCAL surveys of (a) July (0207) and (b) October (0210) 2002, and (c) January/February (0301) and (d) April (0304) 2003. Thick red lines denote the climatological T-S curves, while thin red lines indicate one standard deviation. The green curve represents the mean from the observations (dots) on each graph. Climatologial and cruise mean profiles were obtained using the same stations occupied on each cruise. Figura 3. Diagramas de temperatura-salinidad (T-S) para los cruceros IMECOCAL de (a) julio (0207) y (b) octubre (0210) de 2002 y para (c) enero/febrero (0301) y (d) abril (0304) de 2003. Las líneas gruesas en rojo muestran las climatologías de las curvas T-S y las líneas delgadas en rojo indican una desviación estándar. Las líneas verdes representan la media de todas las observaciones (puntos) para cada gráfico. Los perfiles climatológicos y para cada crucero fueron obtenidos con las mismas estaciones ocupadas en cada crucero.

since data points for the 0207 cruise fall near the climatological mean. The exception for this cruise are warm surface waters $\left(\sigma_{t}<24\right)$, which are not observed in the climatological mean. Starting in October 2002 (fig. 3b), fresher than usual waters are observed for $24.5<\sigma_{t}<25$, while in January (0301) and April (0304) near-surface waters were fresher than the mean profile for $\sigma_{t}<24.6$ and $\sigma_{t}<25.5$, respectively. Besides the difference in salinity, data from October 2002 (fig. 3b) also showed lower than seasonal mean surface temperatures $\left(\sim 2-3^{\circ} \mathrm{C}\right)$. These last estación oceánica ( 2 $\mathrm{m} \mathrm{s}^{-1}$ en IG, fig. 2d). La estación en IG presentó la mayor intensidad y persistencia del viento, con alta variabilidad en la dirección paralela a la costa y velocidades cercanas a la media estacional. La estación en ITS mostró la mayor variabilidad con relación a SQ y BSQ, debido a la influencia de la brisa diurna. La velocidad media del viento (tabla 1) fue máxima en la estación oceánica (IG) y mínima en la estación alejada de la costa (SQ). No se midieron vientos anómalamente débiles, característicos de El Niño, en ninguna 
three cruises showed the presence of less saline water $(\mathrm{S} \sim 33$ ) around the near-surface salinity minimum $\left(\sigma_{\mathrm{t}} \sim 25,0-100 \mathrm{~m}\right)$. This minimum is associated with the CC core, a water mass of subarctic origin. The presence of this relative salinity minimum suggests that during these months, subarctic water entered the region through intensification of equatorward flows or larger spatial extension, or both, of the CC.

Below the $\sigma_{\mathrm{t}}=26$ isopycnal $(200-500 \mathrm{~m})$, mean T-S curves derived from the four cruises are near normal; however, T-S value pairs differing up to two standard deviations from the climatological mean profile are discernible during July, October and January. The presence of more saline waters along $26<\sigma_{\mathrm{t}}<26.5$ suggests a slight increment in the volume of
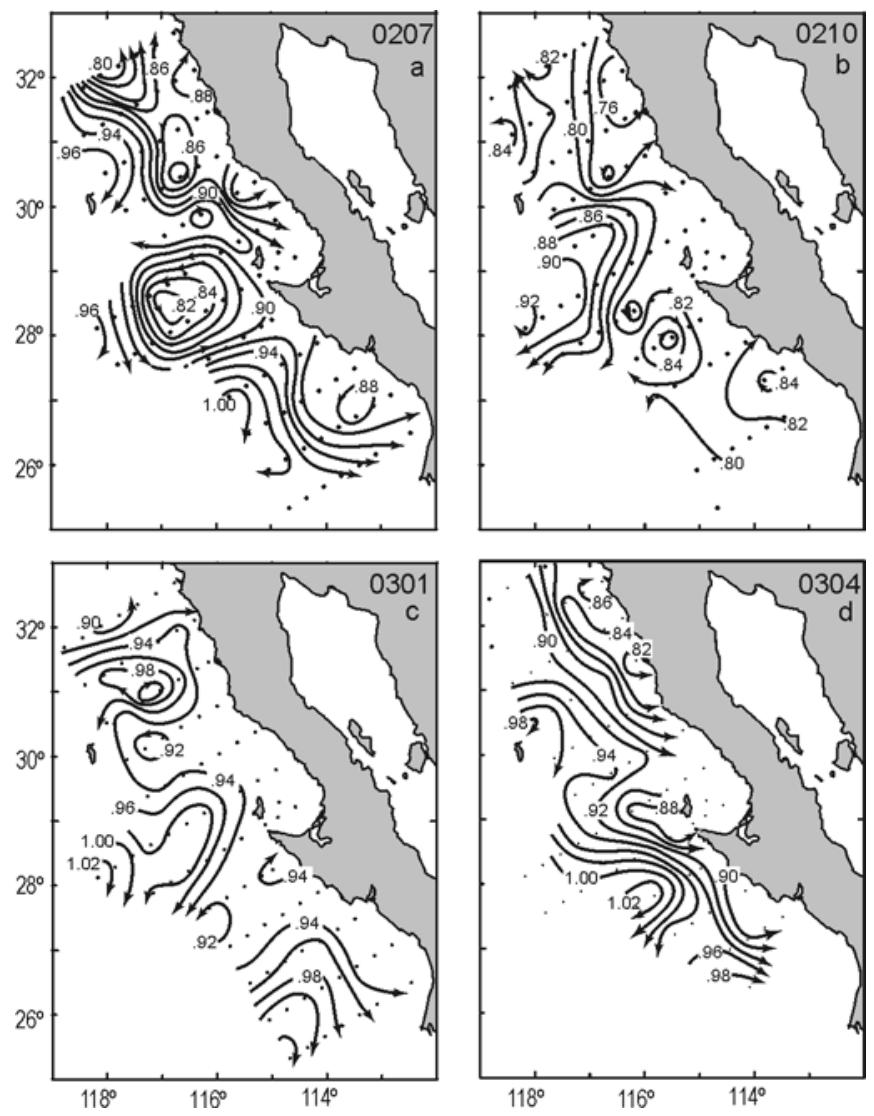

Figure 4. Spatial patterns of upper ocean geostrophic flow estimated from the 0/500 dbar dynamic height field for IMECOCAL cruises: (a) 0207 (12 July to 2 August 2002), (b) 0210 (23 October to 13 November 2002), (c) 0301 (30 January to 20 February 2003) and (d) 0304 (3 to 24 April 2003). The reference level for cruise 0210 was $400 \mathrm{dbar}$; (a), (b) and (c) were taken from Venrick et al. (2003). Contour interval is 0.02 dynamic meters. Current direction is indicated by arrows.

Figura 4. Patrones espaciales del flujo geostrófico superficial estimado del campo de alturas dinámicas 0/500 dbar, para los cruceros IMECOCAL: (a) 0207 (12 de julio a 2 de agosto de 2002), (b) 0210 (23 de octubre a 13 de noviembre de 2002), (c) 0301 (30 de enero a 20 de febrero de 2003) y (d) 0304 (3 a 24 de abril de 2003). El nivel de referencia para el crucero 0210 fue de $400 \mathrm{dbar}$; (a), (b) y (c) fueron tomados de Venrick et al. (2003). El intervalo de contornos es 0.02 metros dinámicos. La dirección de la corriente se indica con flechas. de las estaciones, excepto durante el invierno en IG en eventos que duraron de dos a tres semanas (finales de 2002 e inicios de 2003).

Las series de tiempo de la TSM para IG presentaron un fuerte ciclo estacional, con un máximo relativo $\left(\sim 20^{\circ} \mathrm{C}\right)$ en septiembre y un mínimo $\left(\sim 15^{\circ} \mathrm{C}\right)$ en marzo (fig. 2e). Temperaturas por arriba de la media fueron observadas de enero a septiembre de 2000 y a principios del año 2003.

\section{Características termohalinas}

Se utilizaron diagramas temperatura-salinidad (T-S) para ilustrar las características termohalinas más importantes que prevalecieron durante los muestreos (fig. 3). Para tener una mejor visualización de los cambios en las condiciones oceanográficas, los diagramas T-S fueron contrastados con perfiles climatológicos ( \pm 1 desviación estándar) para cada estación del año, calculados a partir de datos de los programas CalCOFI de 1950-1978 y de IMECOCAL de 1997-2003. Los promedios de temperatura y de salinidad fueron calculados dentro de un intervalo seleccionado de densidad. Las líneas rojas y verdes en las curvas representan el promedio climatológico y de cada crucero, respectivamente. Se tuvo un cuidado especial al comparar los diagramas T-S y los perfiles promedios correspondientes, tomando las mismas estaciones ocupadas durante cada crucero. Las curvas medias por crucero (verde) indican que durante el verano de 2002 persistieron condiciones cercanas a lo normal (fig. 3a), ya que los datos discretos del crucero 0207 se agruparon cerca de la media climatológica. La excepción para este crucero fue el agua cálida superficial $\left(\sigma_{t}<24\right)$, la cual no se observó en la media climatológica. A partir de octubre de 2002 (fig. 3b) se observó agua con menor salinidad que lo usual para el intervalo $24.5<\sigma_{t}<25$, mientras que en enero (0301) y abril (0304) las aguas cercanas a la superficie fueron menos saladas que el perfil promedio para $\sigma_{\mathrm{t}}<24.6 \mathrm{y} \sigma_{\mathrm{t}}$ $<25.5$, respectivamente. Además de las diferencias en salinidad en los datos de octubre de 2002 (fig. 3b), la temperatura superficial mostró también valores menores $\left(\sim 2-3^{\circ} \mathrm{C}\right)$ a los estacionales. Estos tres cruceros tuvieron la presencia de agua con menor salinidad ( $\mathrm{S} \sim 33$ ) cercana al mínimo de salinidad subsuperficial $\left(\sigma_{t} \sim 25,0-100 \mathrm{~m}\right)$. Este mínimo está asociado con el núcleo de la CC, una masa de agua de origen subártico. La presencia del mínimo relativo de salinidad sugiere que durante estos meses el agua del subártico ingresó a la región a través de la intensificación del flujo hacia el ecuador, debido a una mayor extensión de la CC, o como resultado de ambos procesos.

Por debajo de la isopicna $\sigma_{\mathrm{t}}=26(200-500 \mathrm{~m})$, el promedio de las curvas T-S derivado de los cuatro cruceros fue cercano a lo normal; sin embargo, durante julio, octubre y enero fueron muy evidentes las diferencias de más de dos desviaciones estándar entre el perfil climatológico promedio y los valores de T-S. La presencia de agua con mayor salinidad a lo largo de $26<\sigma_{\mathrm{t}}<26.5$ sugiere un ligero incremento en el volumen del 
water carried by the CU between 200 and $500 \mathrm{~m}$ depth (see Durazo and Baumgartner, 2002).

\section{Geostrophic flow}

Dynamic height anomalies at 0 and 200 dbar over the 500 dbar level, except for October 2002 when the length of the hydrographic cable permitted sampling only to $400 \mathrm{~m}$, are used to describe the surface and subsurface fields of flow, respectively (figs. 4, 5). Note that near-surface currents for cruises 0207, 0210 and 0301 have been described in Venrick et al. (2003).

Near-surface dynamic heights depict in general an equatorward flow with considerable mesoscale features (fig. 4a-d). There are indications of poleward flows only at selected coastal locations associated with coastal prominences and upwelling, or near the coast of Punta Eugenia during July 2002 when the northward flow is due to the presence of a cyclonic gyre centered around $200 \mathrm{~km}$ offshore. Thus, the expected northward flow carrying waters from the south, as is usually seen for an El Niño event, was not present.

Below the surface, geostrophic flows at 200 dbar for cruises 0207, 0210 and 0301 (fig. 5a-c) show poleward flows depicting the presence of the $\mathrm{CU}$, adjacent to the coast and the shelf break (July and October 2002) or displaced offshore and circulating around clockwise eddies (January 2003). During the spring of 2003 (fig. 5d), the CU is not clearly discernible in the dynamic height anomaly field.

\section{Temperature and salinity anomalies}

A measure of the changes induced by local and remote forcing during 2002-2003 are temperature and salinity anomaly fields for line 120 (fig. 6), a line that represents the locus of the greatest latitudinal variability of water properties within the CCS. Anomalies are referred to climatological means derived from 33 years of historical data (CalCOFI and IMECOCAL).

The data from summer 2002 (fig. 6a, b) show cores of negative temperature anomalies $\left(\sim-3.5^{\circ} \mathrm{C}\right)$ at about $70 \mathrm{~m}$ depth (fig. 6a) and positive salinity anomalies $(\sim 0.3)$ between 100 and $150 \mathrm{~m}$ depth (fig. 6b). Saltier conditions could indicate El Niño conditions with southern waters being carried northward along the coast. However, as is suggested by the surface and 200 dbar geostrophic currents (figs. 4a, 5a), the cores of colder and saltier water are apparently associated with a cyclonic gyre off Punta Eugenia.

The October 2002 temperature and salinity anomalies (fig. 6c, d) show colder and fresher $\left(-2^{\circ} \mathrm{C},-0.3\right)$ than usual conditions over the upper $100 \mathrm{~m}$. This was reflected in the T-S curves of figure $3 \mathrm{~b}$ as a shifted relative salinity minimum along the $\sigma_{\mathrm{t}} \sim 25$ isopycnal surface. These anomalous conditions suggest that more volume of the CC, that is, an excess of subarctic water, reached the survey region during this season. At around 200 dbar, positive temperature and salinity anomalies for this cruise, as well as the large dispersion of T-S points along the
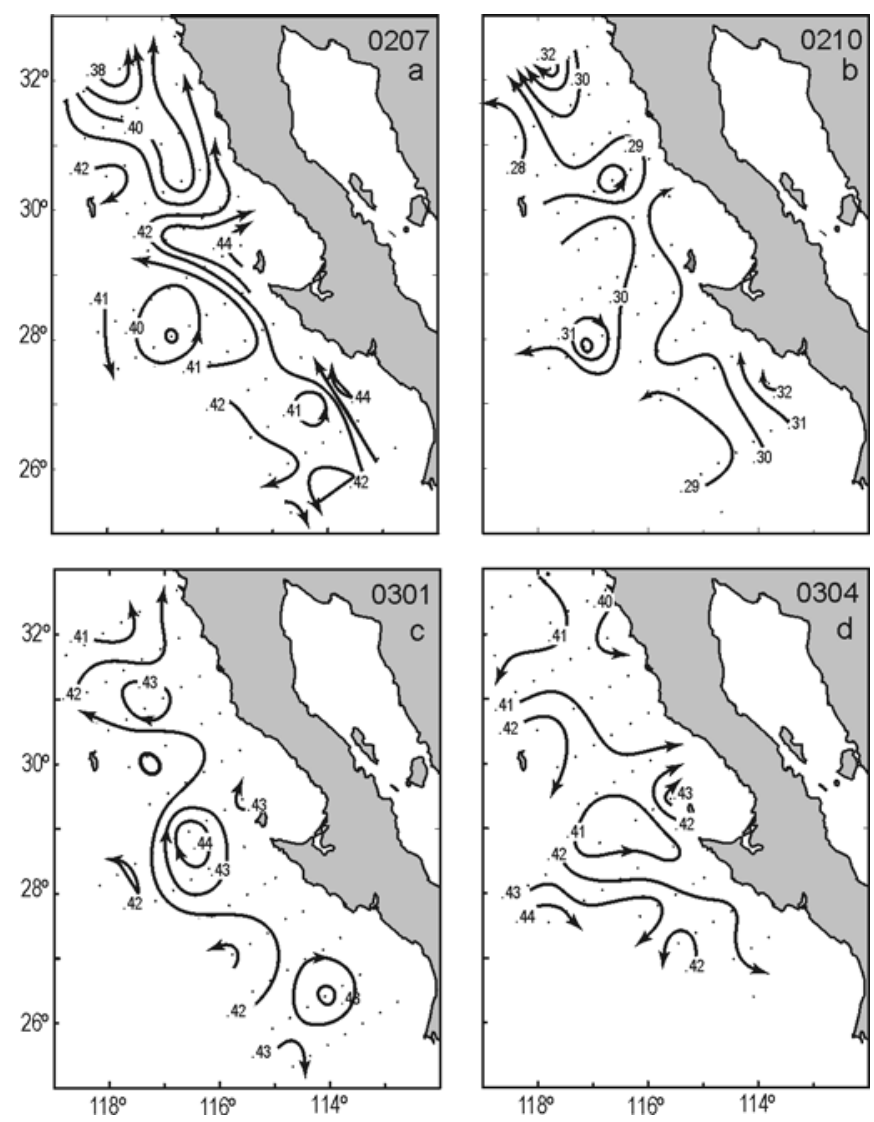

Figure 5. Spatial patterns of the 200-m geostrophic flow estimated from the 200/500 dbar dynamic height field for IMECOCAL cruises: (a) 0207 (12 July to 2 August 2002), (b) 0210 (23 October to 13 November 2002), (c) 0301 (30 January to 20 February 2003) and (d) 0304 (3 to 24 April 2003). The reference level for cruise 0210 was $400 \mathrm{dbar}$. Contour interval is 0.01 dynamic meters. Current direction is indicated by arrows.

Figura 5. Patrones espaciales del flujo geostrófico a 200 m estimado del campo de alturas dinámicas 200/500 dbar para los cruceros IMECOCAL: (a) 0207 (12 de julio a 2 de agosto de 2002), (b) 0210 (23 de octubre a 13 de noviembre de 2002), (c) 0301 (30 de enero a 20 de febrero de 2003) y (d) 0304 (3 a 24 de abril de 2003). El nivel de referencia para el crucero 0210 fue de 400 dbar. El intervalo de contornos es 0.01 metros dinámicos. La dirección de la corriente se indica con flechas.

agua transportada por la CU entre 200 y $500 \mathrm{~m}$ de profundidad (ver Durazo y Baumgartner, 2002).

\section{Flujo geostrófico}

Las anomalías de altura dinámica a 0 y 200 dbar sobre un nivel de referencia de 500 dbar, excepto para octubre de 2002 cuando la longitud del cable hidrográfico permitió solamente llegar hasta los $400 \mathrm{~m}$, fueron utilizadas para describir los campos de los flujos superficiales y subsuperficiales, respectivamente (figs. 4, 5). Las corrientes cercanas a la superficie para los cruceros 0207, 0210 y 0301 han sido previamente explicadas en Venrick et al. (2003).

Las alturas dinámicas cercanas a la superficie mostraron en general un flujo hacia el ecuador, con considerables estructuras de mesoescala (fig. 4a-d). Se presentaron indicaciones de 

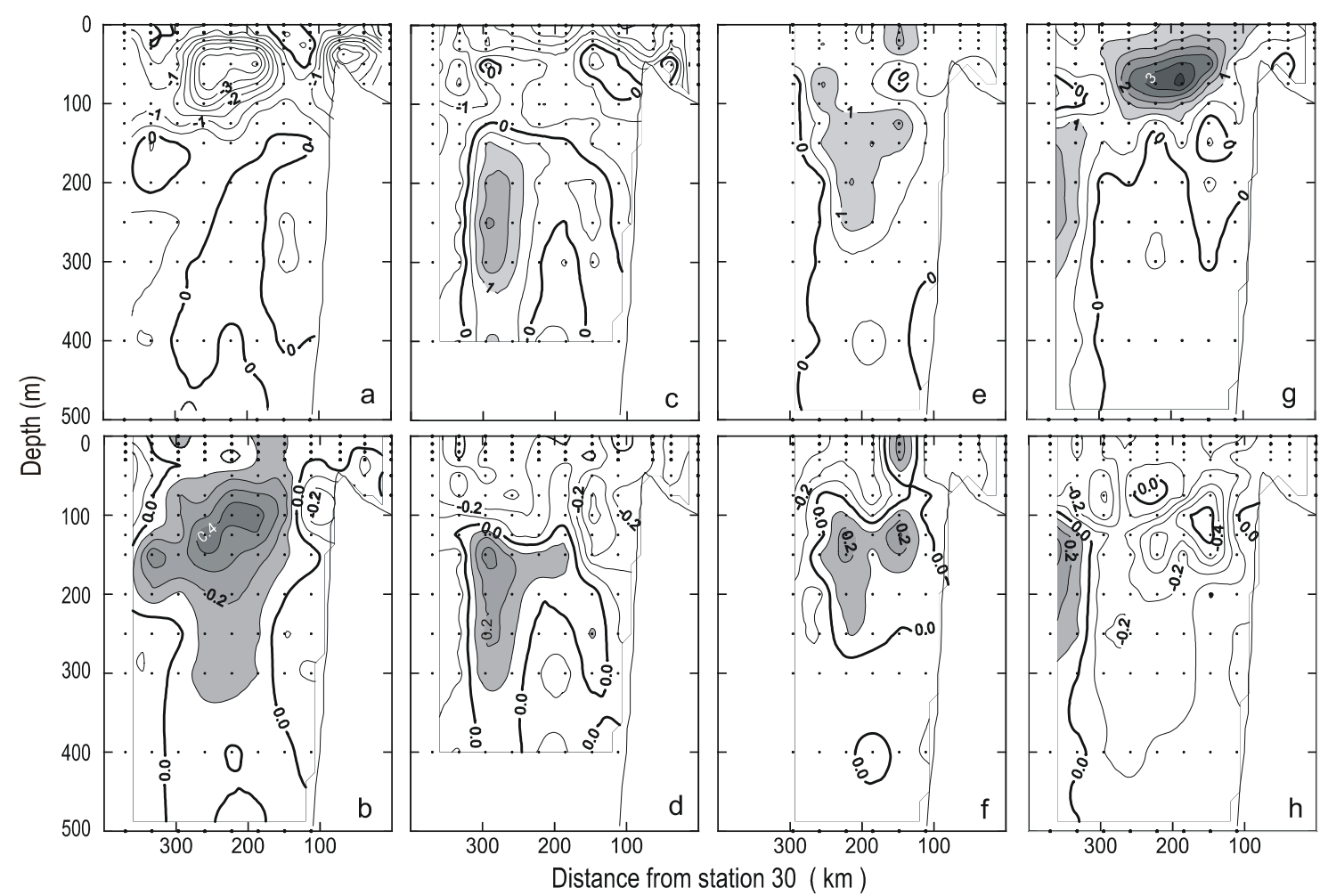

Figure 6. Temperature $\left(\mathbf{a}, \mathbf{c}, \mathbf{e}, \mathbf{g} ;{ }^{\circ} \mathbf{C}\right)$ and salinity anomalies $(\mathbf{b}, \mathbf{d}, \mathbf{f}, \mathbf{h})$ for cruises $0207,0210,0301$ and 0304 along line 120 . Shaded areas represent positive temperature and salinity anomalies larger than $1^{\circ} \mathrm{C}$ and 0.1 , respectively. Anomalies are referred to the climatological mean computed using CalCOFI (1950-1978) and IMECOCAL (1997-2003) data sets.

Figura 6. Anomalías de temperatura $\left(\mathbf{a}, \mathbf{c}, \mathbf{e}, \mathbf{g} ;{ }^{\circ} \mathrm{C}\right)$ y salinidad $(\mathbf{b}, \mathbf{d}, \mathbf{f}, \mathbf{h})$ para los cruceros 0207, 0210, 0301 y 0304 a lo largo de la línea 120. Las áreas sombreadas representan anomalías positivas de temperatura y de salinidad mayores a $1^{\circ} \mathrm{C}$ y 0.1 , respectivamente. Las anomalías fueron referidas al promedio climatológico a partir de los datos de los progamas CalCOFI (1950-1978) e IMECOCAL (1997-2003).

$\sigma_{t}=26$ in figure 3b, suggest an increment of the volume of water of equatorial origin carried by the CU (fig. 5b).

Small temperature anomalies $\left(\sim 1^{\circ} \mathrm{C}\right)$ during January 2003 (fig. 6e) depict near-normal conditions, while near-surface salinity values lower than the mean $(\sim-0.3$, fig. $6 f)$ suggest that a greater volume of subarctic waters encroached upon the Baja California survey region. As in the October cruise, this is substantiated by the shifting in the T-S diagrams towards lower salinities above the $\sigma_{t}>25.3$ (fig. 3c). Below $100 \mathrm{~m}$ depth, positive temperature and salinity anomalies $\left(1^{\circ} \mathrm{C}, 0.2\right)$ suggest, as in cruise 0207, an enhancement of flow of the equatorial waters (CU, fig. 5c).

Finally, the April 2003 (0304) data depicted warmer than usual waters $\left(\sim 3^{\circ} \mathrm{C}\right.$, fig. $\left.6 \mathrm{~g}\right)$ in a core centered at $70 \mathrm{~m}$ depth. As suggested by the near-surface geostrophic currents of figure 4 , this anomalous warming could be of local origin. This can result from surface waters being transported into Vizcaíno Bay, recirculated inside in an almost permanent clockwise gyre (Palacios-Hernández et al., 1996) and later transported outside (warmer), to be incorporated again into the southern flow of the CC (fig. 4). Lower than normal (-0.4 to 0 ) salinities were found throughout most of the section, but they were largest $(\sim-0.4)$ in a subsurface core centered between 100 and $150 \mathrm{~m}$ adjacent to the shelf break (fig. 6h). The T-S flujos hacia el polo solamente en algunas localidades costeras, asociados con la geomorfología de la costa y con afloramientos locales. También fue evidente un flujo hacia el polo en julio de 2002 cerca de la costa de Punta Eugenia, debido a la presencia de un giro ciclónico centrado alrededor de $200 \mathrm{~km}$ fuera de la costa. Por lo tanto, no estuvo presente el flujo hacia el norte que transporta aguas desde el sur característico de eventos El Niño.

Por debajo de la superficie, la velocidad geostrófica a 200 dbar para los cruceros 0207, 0210 y 0301 (fig. 5a-c) mostró flujos hacia el polo, lo que indicó la presencia de la CU, ya sea adyacente a la costa y a la pendiente de la plataforma continental (julio y octubre de 2002), o desplazada hacia fuera de la costa y circulando alrededor de remolinos anticiclónicos (enero de 2003). Durante la primavera de 2003 (fig. 5d) la CU no fue claramente evidente en el campo de la anomalía de altura dinámica.

\section{Anomalías de temperatura y salinidad}

Una medida de los cambios inducidos por el forzamiento local y el remoto producidos durante 2002-2003 son los campos de las anomalías de temperatura y salinidad calculadas para la línea 120 (fig. 6). Esta línea representa el lugar donde se 

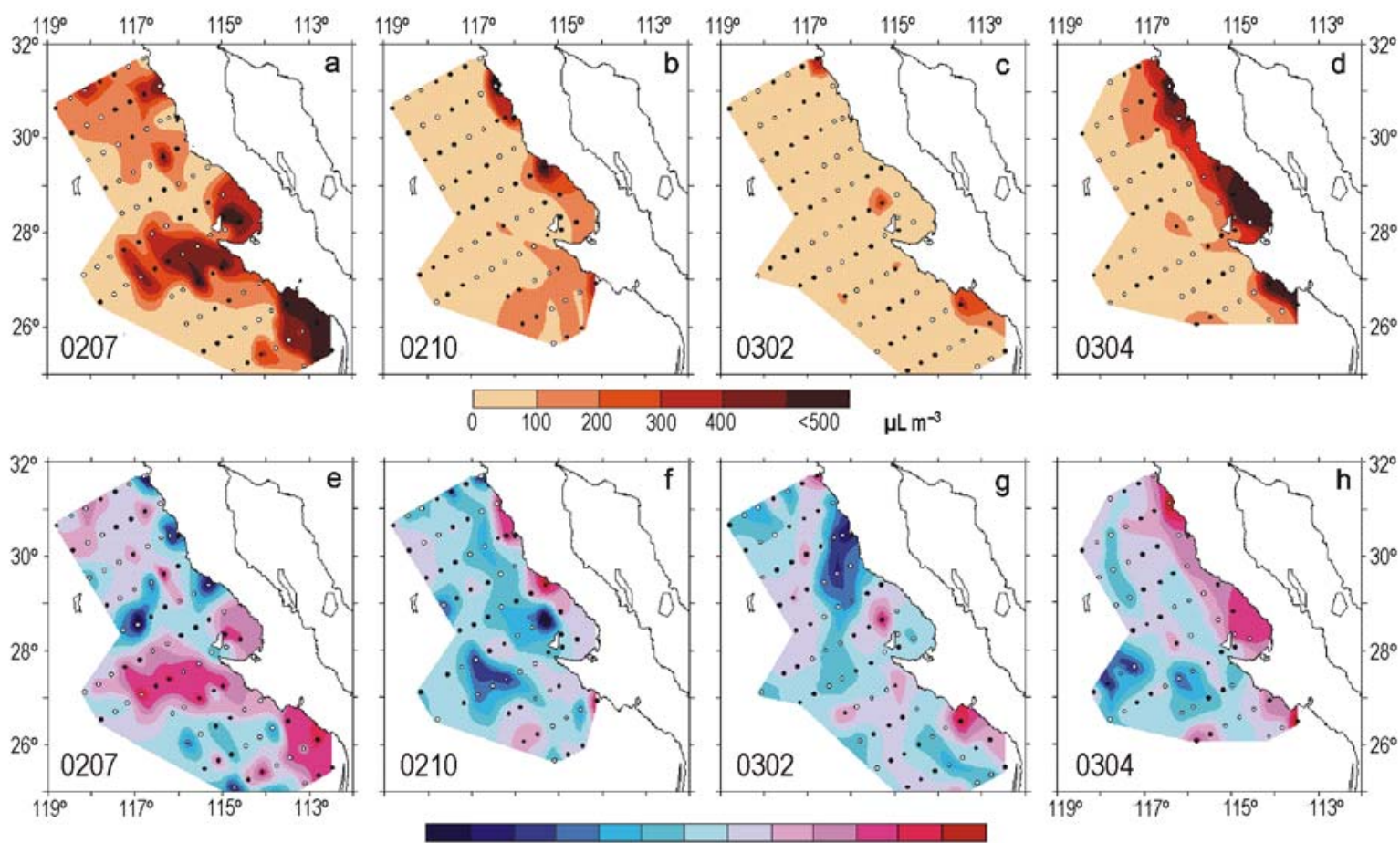

$\begin{array}{llllllllllllll}-1.2 & -1 & -0.8 & -0.6 & -0.4 & -0.2 & 0 & 0.2 & 0.4 & 0.6 & 0.8 & 1 & \log \left(\mu \mathrm{L}^{-3}\right)\end{array}$

Figure 7. Zooplankton biomass distribution (a-d) and zooplankton biomass anomalies (e-h) during July and October 2002, and February and April 2003. For each cruise, anomalies were computed by removing the seasonal mean for the period 1951-1984, after transforming data to logarithms. Station sampling during light (o) and dark (•) hours.

Figura 7. Distribución de la biomasa del zooplancton (a-d) y anomalías de la biomasa del zooplancton (e-h) durante julio y octubre de 2002, y febrero y abril de 2003. Para cada crucero las anomalías fueron calculadas removiendo la media estacional del periodo 19511984, después de transformar los datos a logaritmos. Estaciones muestreadas durante horas de luz (০) y oscuridad (•).

diagram (fig. 3d) and the geostrophic velocities depicting equatorward flow and absence of poleward flows (CU) at $200 \mathrm{~m}$ (figs. 4, 5d), indicate that these waters come from a northern source, namely the subarctic.

\section{Biological patterns}

The July 2002 cruise (0207) exhibited high zooplankton biomass $\left(100-500 \mu \mathrm{L} \mathrm{m}^{-3}\right)$ over most of the survey area (fig. $7 \mathrm{a})$, separated by two zonal bands of lower biomass $(<100 \mu \mathrm{L}$ $\left.\mathrm{m}^{-3}\right)$. In the Gulf of Ulloa $\left(\sim 24.5-26.5^{\circ} \mathrm{N}\right)$ even higher biomass was found $\left(>500 \mu \mathrm{L} \mathrm{m}^{-3}\right)$. Zooplankton biomass anomalies during cruise 0207 (logarithms, fig. 7e) were highly variable from one sampled station to another; however, a negative tendency was evident in the coastal zone off northern Baja California and a positive one in the central lines and the Gulf of Ulloa.

Phytoplankton chlorophyll $a$ in summer 2002 was high $\left(>1.0 \mathrm{mg} \mathrm{m}^{-3}\right.$, fig. 8) near the coastal locations of lines 100 and 120 (fig. 8a, e). These low chlorophyll concentrations are associated with warm and low nutrient waters recirculated by the Southern California Bight Eddy (Lynn and Simpson, 1987). Nearshore locations with the highest chlorophyll were related to 10 -m water with temperature below $15^{\circ} \mathrm{C}$ (Venrick et al., 2003), indicating that coastal upwelling processes during this exhibe la mayor variabilidad estacional en las propiedades del agua del SCC. Las anomalías fueron referidas a medias climatológicas derivadas de 33 años de datos históricos (CalCOFI e IMECOCAL).

Los datos para el verano de 2002 (fig. 6a, b) mostraron núcleos de anomalías negativas en la temperatura $\left(\sim-3.5^{\circ} \mathrm{C}\right)$ cercanos a los $70 \mathrm{~m}$ de profundidad (fig. 6a) y anomalías positivas de salinidad ( 0.3) entre 100 y $150 \mathrm{~m}$ de profundidad (fig. 6b). El agua con mayor salinidad indica la presencia de condiciones El Niño cuando las aguas sureñas son transportadas hacia el norte a lo largo de la costa. Sin embargo, como es sugerido por las corrientes geostróficas calculadas para la superficie y a $200 \mathrm{db}$ (figs. 4a, 5a), los núcleos de agua fría y más salada fueron aparentemente asociados con un giro ciclónico frente a Punta Eugenia.

En octubre de 2003 las anomalías de temperatura y de salinidad en los $100 \mathrm{~m}$ superiores (fig. 6c, d) evidenciaron la presencia de aguas más frías y con menor salinidad $\left(-2^{\circ} \mathrm{C}\right.$, -0.3) que lo normal. Esto también se reflejó en los diagramas T-S de la figura 3b, ya que hubo un cambio en el mínimo relativo de salinidad a lo largo de la isopicna $\sigma_{t} \sim 25$. Estas condiciones anómalas sugieren la entrada a la región de estudio de un mayor volumen de agua de la CC en esa estación del año, considerándose como un exceso de agua de origen subártico. Cerca de los 200 dbar se presentaron anomalías positivas de 


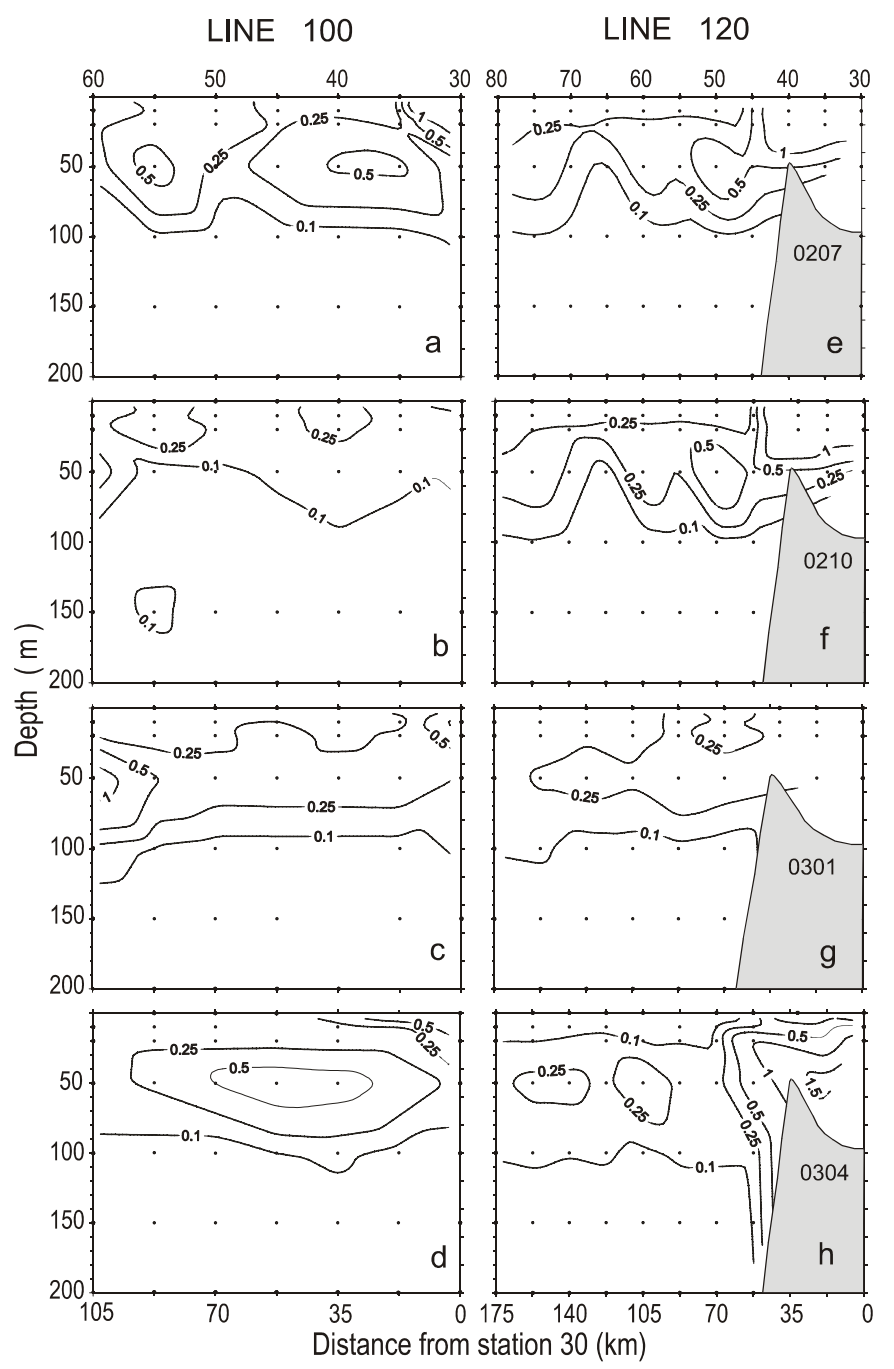

Figure 8. Vertical distribution of chlorophyll a $\left(\mathrm{mg} \mathrm{m}^{-3}\right)$ for lines 100 (a-d) and $120(\mathbf{e}-\mathbf{h})$ for cruises 0207, 0210, 0301 and 0304. Note the different horizontal scales. Numbers on top represent the station number along each line.

Figura 8. Distribución vertical de la clorofila a $\left(\mathrm{mg} \mathrm{m}^{-3}\right)$ en las líneas 100 (a-d) y 120 (e-h) de los cruceros 0207, 02010, 0301 y 0304. Note las diferencias en las escalas horizontales. Los números en la parte superior representan la estación a lo largo de cada línea.

survey increased phytoplankton production. High chlorophyll concentrations extending offshore in line 120 (fig. 8e) appear to be related to nutrient-rich waters advected from the coast and circulated through a cyclonic gyre off Punta Eugenia.

Zooplankton biomass decreased during the autumn survey (0210, fig. 7b), with volumes lower than $100 \mu \mathrm{L} \mathrm{m}^{-3}$ over most of the IMECOCAL grid. It remained slightly higher at some coastal locations and offshore south of Punta Eugenia. Logarithmic anomalies were negative in most of the area, indicating a season less rich than the historic mean (fig. 7f). Generally, low phytoplankton biomass (fig. 8) was related to decreased coastal upwelling and increased near-surface temperatures (Venrick et al., 2003). High concentrations of chlorophyll $\left(>1.0 \mathrm{mg} \mathrm{m}^{-3}\right.$ ) were only located inside Vizcaíno Bay, and at temperatura y salinidad para el crucero de octubre de 2003, así como una gran dispersión de los puntos T-S a lo largo de la $\sigma_{t}=26$ en la figura 3b. Esto sugiere un incremento en el volumen de agua de origen ecuatorial, transportada por la CU (fig. 5b).

Las bajas anomalías de la temperatura $\left(\sim 1^{\circ} \mathrm{C}\right)$ durante enero de 2003 (fig. 6e) mostraron una condición cercana a la normal, mientras que los valores de salinidad por debajo del promedio cercanos a la superficie $(\sim-0.3$, fig. $6 f)$ sugieren que una mayor cantidad de agua del subártico invadió la región de muestreo frente a Baja California. Así como sucedió en el crucero de octubre, hubo un cambio substancial en el diagrama T-S hacia salinidades menores sobre la $\sigma_{\mathrm{t}}>25.3$ (fig. 3c). Por debajo de los $100 \mathrm{~m}$ de profundidad las anomalías positivas de temperatura y salinidad $\left(1^{\circ} \mathrm{C}, 0.2\right)$ sugieren un enriquecimiento del flujo del agua ecuatorial (CU, fig. 5c), como sucedió en el crucero 0207.

Finalmente, los datos de abril de 2003 (0304) mostraron aguas más cálidas que lo usual $\left(\sim 3^{\circ} \mathrm{C}\right.$, fig. $\left.6 \mathrm{~g}\right)$, en un núcleo centrado a $70 \mathrm{~m}$ de profundidad. Como fue sugerido por el flujo geostrófico cercano a la superficie de la figura 4, el calentamiento anómalo podría ser de origen local. Esto pudo ser originado por el transporte de aguas superficiales hacia el interior de Bahía Vizcaíno, las cuales fueron recirculadas dentro de la bahía en forma de un giro anticiclónico casi permanente (Palacios-Hernández et al., 1996) y posteriormente transportadas hacia fuera (más calientes) para ser incorporadas de nuevo en el flujo hacia el sur de la CC (fig. 4). Las salinidades menores a lo normal (-0.4-0) fueron medidas en la mayor parte de esta sección, pero fueron mucho mayores $(\sim-0.4)$ en un núcleo subsuperficial centrado entre 100 y $150 \mathrm{~m}$, adyacente a la pendiente de la plataforma (fig. 6h). Los diagramas T-S (fig. 3d) y las velocidades geostróficas mostraron un flujo hacia el ecuador y la ausencia de flujos hacia el polo (CU) a $200 \mathrm{~m}$ de profundidad (figs. 4, 5d), lo que indica que estas aguas vinieron de una fuente norteña, es decir del subártico.

\section{Patrones biológicos}

Los datos del crucero de julio de 2002 (0207) mostraron alta biomasa del zooplancton (100-500 $\mu \mathrm{L} \mathrm{m}^{-3}$ ) en la mayor parte del área muestreada (fig. 7a), separada por dos zonas de muy baja biomasa $\left(<100 \mu \mathrm{L} \mathrm{m}^{-3}\right)$. En el Golfo de Ulloa $\left(\sim 24.5-26.5^{\circ} \mathrm{N}\right)$ se encontró también muy alta biomasa $(>500$ $\mu \mathrm{L} \mathrm{m}^{-3}$ ). Las anomalías en la biomasa del zooplancton calculadas para el crucero 0207 (logaritmos, fig. 7e) fueron altamente variables de una localidad a otra; sin embargo, se obtuvo una notoria tendencia negativa en la zona costera del norte de Baja California y una tendencia positiva en las líneas centrales y en el Golfo de Ulloa.

En el verano de 2002 la clorofila $a$ del fitoplancton fue alta (>1.0 $\mathrm{mg} \mathrm{m}^{-3}$ ) cerca de las estaciones costeras de la línea $100 \mathrm{y}$ 120 (fig. 8a, e). Las bajas concentraciones de clorofila fueron asociadas con aguas cálidas y con baja concentración de nutrientes recirculadas por el giro del la Ensenada del Sur de 
North Baja California (lines 100-110)
North Baja California (lines 113-133)

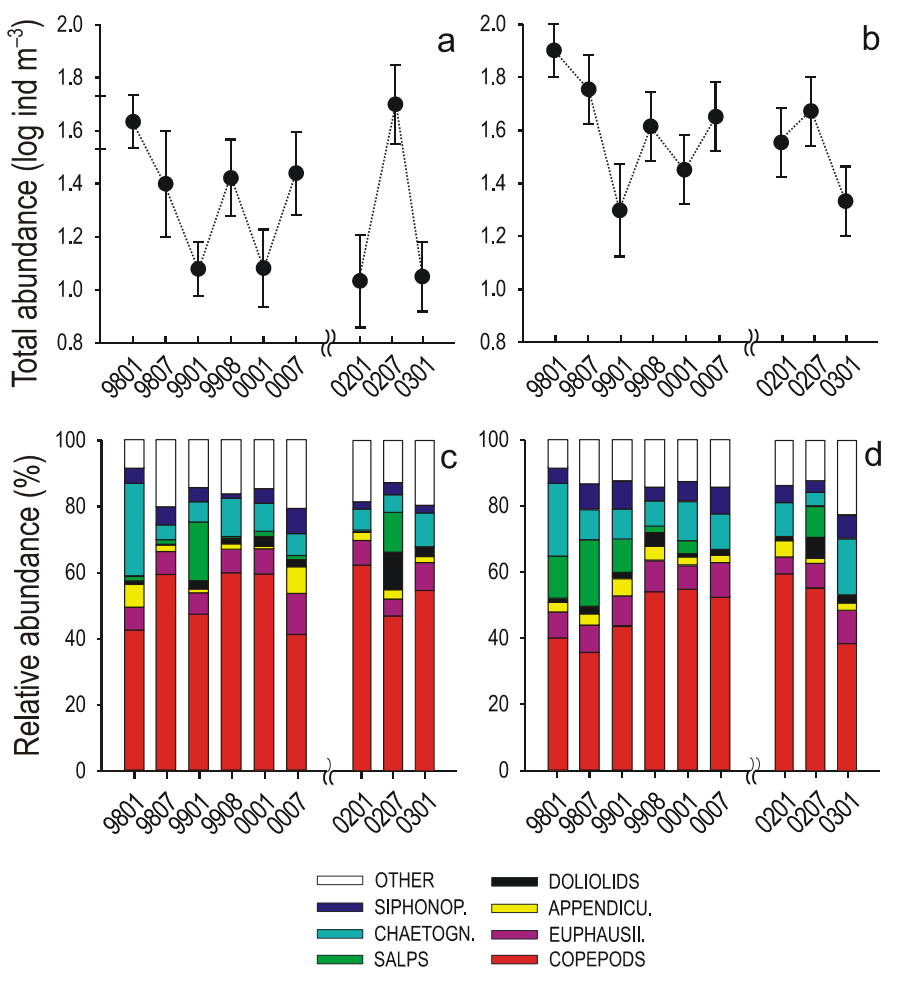

Figure 9. Mean total zooplankton abundance $(\mathbf{a}, \mathbf{b})$ and mean relative abundance of the main zooplankton taxa $(\mathbf{c}, \mathbf{d})$ during winter and summer of 1998-2000 and 2002-2003. Bars indicate 95\% confidence intervals. Means were computed from data for the northern $(a, c)$ and central $(b, d)$ regions off Baja California.

Figura 9. Promedios de la abundancia total del zooplancton $(\mathbf{a}, \mathbf{b})$ y de la abundancia relativa de los principales taxones del zooplancton (c, d) durante el invierno y verano de 1998-2000 y 2002-2003. Las barras muestran los intervalos de confianza al 95\%. Las medias fueron calculadas de datos de las regiones norte $(a, c)$ y central $(b, d)$ frente a Baja California.

one inshore station of line 120 (fig. 8f). For most of the sampled area phytoplankton chlorophyll was below $0.25 \mathrm{mg} \mathrm{m}^{-3}$, with high concentrations only at nearshore stations of line 120 .

Winter (0301) zooplankton biomass was extremely low (fig. 7c), with values less than $100 \mu \mathrm{L} \mathrm{m}^{-3}$ over most of the region; however, the maximum negative anomalies were found toward the north (fig. 7g). Chlorophyll concentrations were also very low, with values $>1.0 \mathrm{mg} \mathrm{m}^{-3}$ only in offshore subsurface waters of line 100 (fig. 8c). The 10-m temperature had a north-south gradient (see Venrick et al., 2003), which indicated that coastal upwelling processes were absent or very weak during this period. Phytoplankton biomass showed a typical condition of winter season, with low near-surface values and small vertical changes in the water column.

Zooplankton volumes along the coast rebounded during April 2003 (fig. 7d), surpassing $500 \mu \mathrm{L} \mathrm{m}^{-3}$ in coastal prominences such as Punta Colonet $\left(\sim 30.8^{\circ} \mathrm{N}\right)$, Vizcaíno Bay and Punta Abreojos $\left(\sim 26.5^{\circ} \mathrm{N}\right)$. This recovery appears to be due to the increased phytoplankton biomass (fig. 8d). Zooplankton
California (Lynn y Simpson, 1987). Las mayores concentraciones de clorofila en las estaciones cercanas a la costa estuvieron relacionadas con temperatura a $10 \mathrm{~m}$ de profundidad por debajo de $15^{\circ} \mathrm{C}$ (Venrick et al., 2003), indicando que los afloramientos costeros incrementaron la producción del fitoplancton en este crucero. Las altas concentraciones de clorofila se extendieron hacia fuera de la costa en las líneas 120 (fig. 8e), aparentemente asociadas con aguas ricas en nutrientes provenientes de la costa y circuladas a través de un giro ciclónico frente a Punta Eugenia.

La biomasa del zooplancton disminuyó durante el crucero de otoño (0210, fig. 7b), con volúmenes menores a $100 \mu \mathrm{L} \mathrm{m}^{-3}$ en la mayor parte de la red de muestro del IMECOCAL. Esta biomasa permaneció ligeramente alta en algunas localidades costeras y fuera de la costa hacia el sur de Punta Eugenia. Las anomalías logarítmicas fueron negativas en gran parte del área de estudio, indicando una estación del año menos rica que la media histórica (fig. 7f). Generalmente la baja biomasa del fitoplancton (fig. 8) estuvo relacionada con el decremento en la intensidad de los afloramientos costeros y con el incremento de la temperatura cercana a la superficie (Venrick et al., 2003). Solamente se localizaron altas concentraciones de clorofila (>1.0 $\mathrm{mg} \mathrm{m}^{-3}$ ) dentro de Bahía Vizcaíno y en una localidad costera de la línea 120 (fig. 8f). La clorofila del fitoplancton estuvo por abajo de $0.25 \mathrm{mg} \mathrm{m}^{-3}$ en gran parte del área muestreada, con altas concentraciones solamente en las estaciones costeras de la línea 120.

Durante el invierno (0301) la biomasa del zooplancton fue extremadamente baja (fig. 7c), con valores menores a $100 \mu \mathrm{L}$ $\mathrm{m}^{-3}$ en la mayor parte de la región de estudio. Sin embargo, las anomalías negativas máximas fueron encontradas hacia el norte (fig. 7g). La concentración de la clorofila fue también muy baja en este periodo, con valores $>1.0 \mathrm{mg} \mathrm{m}^{-3}$ solamente en aguas subsuperficiales alejadas de la costa de la línea 100 (fig. 8c). La temperatura a $10 \mathrm{~m}$ tuvo un marcado gradiente norte-sur (ver Venrick et al., 2003), lo cual indica que los procesos de afloramientos costeros no se presentaron o fueron muy débiles durante esta estación del año. La biomasa del fitoplancton mostró una condición típica del invierno, con bajos valores cercanos a la superficie y ligeros cambios con la profundidad en la columna de agua.

Los altos volúmenes del zooplancton a lo largo de la costa se acrecentaron durante abril de 2003 (fig. 7d) excediendo los $500 \mu \mathrm{L} \mathrm{m}^{-3}$ en algunas regiones costeras como Punta Colonet $\left(\sim 30.8^{\circ} \mathrm{N}\right)$, Bahía Vizcaíno y Punta Abreojos ( 26.5ํN). Esta recuperación parece ser debida al incremento en la biomasa del fitoplancton (fig. 8d). Las anomalías positivas en el zooplancton (fig. 7h) indican que este aumento en el zooplancton de la zona costera para la primavera de 2003 fue mayor que lo usual. Las concentraciones de clorofila fueron altas (fig. 8d, h) principalmente en las estaciones costeras, asociadas con un incremento en el esfuerzo del viento (fig. 2d) y del flujo hacia el ecuador (fig. 4 ).

La abundancia total y la estructura del zooplancton para las regiones norte y central son mostradas en la figura 9. Las 
positive anomalies (fig. 7h) indicate that this rebound over the coastal zone was higher than usual for spring 2003. Chlorophyll concentrations were high (fig. 8d, h), mainly at inshore locations associated with an increase of wind stress (fig. 2d) and equatorward flow (fig. 4).

Total zooplankton abundance and structure for northern and central Baja California are depicted in figure 9. Abundances for summer (0207) and winter (0301) (fig. 9a, b) follow the tendency observed in biomass, with high values in July 2002 and low values in February 2003. Total abundance was similar in the northern and central zones during the 0207 cruise (antilogged mean $=49$ ind $\mathrm{m}^{-3}$ ), whereas in 0301 it was lower in the northern than in the central region (antilogged means $=10$ and 22 ind $\mathrm{m}^{-3}$, respectively). The seasonal change was similar to that observed in previous summer to winter transitions for the period 1999-2000. Winter 1998 was anomalous, displaying abundances in the range usually observed during the summer, while in other winters they were low (fig. 9a, b).

The zooplankton structure did not show clear seasonal differences, but there were interannual and regional differences (fig. 9c, d). While copepods usually occupy $50-60 \%$ of the zooplankton abundance in the central region, they represented only 36-44\% during winter 1998 and its transition to La Niña in 1999, due mainly to the presence of salp blooms (fig. 9d). In the northern region, salps were abundant only in January 1999 and July 2002 (fig. 9c). A high percentage of chaetognaths was a feature of winter 1998, observed again in February 2003 (mainly in the central region), and could be related to the weak El Niño conditions. The larger size of salps, compared to chaetognaths, could explain the main differences in biomass between both cruises. The high percentage of salps found in July 2002 was also observed in July 1998, although restricted to the central region. These regional differences could be related to the kind of species present in each case. For example, Thalia orientalis was dominant during cruise 9807 (Hereu et al., 2003), while the large Pegea socia was very abundant in 0207.

\section{Discussion}

Near-surface thermohaline data, the absence of poleward coastal flow and the typical northwesterly winds reported here, do not indicate the presence of an El Niño during 2002-2003, as has been defined from previous events, e.g. 1997-1998 (Lynn et al., 1998; Hayward et al., 1999; Bograd et al., 2000; Durazo and Baumgartner, 2002; Lynn and Bograd, 2002). The T-S relationships indicated that waters above $25.5 \sigma_{\mathrm{t}}(0-100 \mathrm{~m})$ had lower salinity than the climatological mean for most of the cruises. Low-salinity waters were observed near the surface from July 2002 through April 2003. The lowest salinity values (S 33) were measured in October 2002 and January 2003. Only during October 2002 were these low salinity waters associated with negative temperature anomalies. Lower than the mean salinities may be seen as a logical consequence of stronger than usual equatorward winds that transport more volume abundancias para el verano (0207) e invierno (0301) (fig. 9a, b) siguieron la tendencia observada en la biomasa, con valores altos en julio de 2002 y bajos en febrero de 2003. La abundancia total durante el crucero 0207 fue similar en las zonas norte $\mathrm{y}$ central (promedio antilog $=49$ ind $\mathrm{m}^{-3}$ ), mientras que en 0301 ésta fue menor en la región norte que la central (promedios antilog $=10$ y 22 ind $\mathrm{m}^{-3}$, respectivamente). El cambio estacional fue similar al observado previamente durante la transición verano-invierno en el periodo 1999-2000. El invierno de 1998 fue un periodo anómalo, con altas abundancias dentro del intervalo usualmente observado durante el verano, mientras que en otros inviernos se obtuvieron bajas abundancias (fig. 9a, b).

La estructura del zooplancton no mostró una estricta diferencia estacional, pero sí tuvo diferencias interanuales y regionales (fig. 9c, d). Los copépodos usualmente ocupan entre $50 \%$ y $60 \%$ de la abundancia total del zooplancton de la región central; éstos representaron solamente entre $36 \%$ y $44 \%$ durante el invierno de 1998 y la transición hacia La Niña en 1999, debido principalmente a la presencia de los florecimientos de salpas (fig. 9d). En la región norteña las salpas fueron abundantes solamente en enero de 1999 y en julio de 2002 (fig. 9c). Un alto porcentaje de quetognatos fue la característica más sobresaliente en invierno de 1998, siendo observados de nuevo en febrero de 2003 (principalmente en la región central), lo que podría estar relacionado con una débil condición El Niño. El gran tamaño de las salpas, comparado al de los quetognatos, podría explicar la principal diferencia en biomasa entre ambos cruceros. El alto porcentaje de salpas encontrado en julio de 2002 fue también observado en julio de 1998, aunque restringido a la región central. Estas diferencias regionales podrían estar relacionadas con el tipo de especies presentes en cada uno de los casos. Por ejemplo, Thalia orientalis fue dominante durante el crucero 9807 (Hereu et al., 2003), mientras que la especie de gran tamaño Pegea socia fue muy abundante en el crucero 0207.

\section{Discusión}

La estructura termohalina cercana a la superficie, la ausencia del flujo costero hacia el polo y el comportamiento de los vientos del noroeste observados durante el periodo, no evidencian la presencia de un evento El Niño frente a Baja California durante el periodo 2002-2003, tal y como fue definido para los eventos previos, por ejemplo el de 1997-1998 (Lynn et al., 1998; Hayward et al., 1999; Bograd et al., 2000; Durazo y Baumgartner, 2002; Lynn y Bograd, 2002). Las relaciones T-S indicaron que el agua por arriba de $\sigma_{\mathrm{t}}=25.5(0-100 \mathrm{~m})$ para la mayoría de los cruceros tuvo una menor salinidad que el promedio climatológico. El agua con baja salinidad fue detectada cerca de la superficie desde julio de 2002 hasta abril de 2003. Los valores más bajos de salinidad ( $\mathrm{S} \sim 33$ ) se midieron en octubre de 2002 y en enero de 2003. Solamente durante octubre de 2002 estas aguas de menor salinidad se asociaron con anomalías negativas de temperatura. Se puede considerar que 
of water of northern origin. More intense winds are the typical conditions of a La Niña event. However, the wind records presented above and the T-S diagrams for 2002-2003 indicated that the atmospheric and hydrographic conditions were substantially different than those reported for La Niña 1999-2000 (Durazo et al., 2001; Durazo and Baumgartner, 2002). During La Niña, increased upwelling rises the nutricline in the coastal region of Baja California (Espinosa-Carreón et al., 2004) and brings higher salinity waters to the surface; therefore, the relative salinity minimum between 25 and $25.5 \sigma_{t}$, signaling the core of the CC, is not as evident (Durazo and Baumgartner, 2002).

Subarctic water is the source of low salinity in the northeastern Pacific (Lynn and Simpson, 1987), and it has been influencing the CCS since the summer of 2002 (Huyer, 2003; Kosro, 2003; Venrick et al., 2003). The results shown here suggest that the subarctic water enhancement observed at northern latitudes, reached as far south as $28^{\circ} \mathrm{N}$ during the period from July 2002 to April 2003. In consequence, the weak El Niño 2002-2003 could have been concealed by the subarctic influence.

Below approximately $100 \mathrm{~m}$ depth, a well-developed CU and the dispersion of T-S data towards higher salinities along the 26-26.5 $\sigma_{\mathrm{t}}$ suggested that El Niño was present from July 2002 to January 2003. The timing of the positive anomalies observed differs from the findings of Venrick et al. (2003), who reported a moderate El Niño over southern California waters only during early 2003. When compared to anomalies recorded during the 1997-1998 El Niño event, the magnitude of the temperature and salinity anomalies $\left(1-2^{\circ} \mathrm{C}, 0.2-0.4\right)$ indicated that its effect was weak.

The chlorophyll $a$ data also substantiate the absence of surface warming associated with El Niño conditions. Even April 2003 showed increased phytoplankton biomass at coastal locations, two-fold higher than those reported by Lavaniegos et al. (2003) for waters off Baja California during the 1997-1998 El Niño event. The increased chlorophyll levels in April 2003 could also be interpreted as the end of El Niño, since January/ February 2003 reported almost normal conditions for the eastern and central equatorial Pacific, and by May 2003 the anomalous warm conditions had completely dissipated (http:// www.cpc.ncep.noaa.gov; McPhaden, 2004).

Changes in zooplankton biomass followed a similar pattern, responding to the increase in chlorophyll with anomalously high values in nearshore stations; however, the situation was conspicuously different to that observed during the previous strong El Niño 1997-1998 in Baja California (Lavaniegos et al., 2002). The zooplankton biomass maintained a typical level during autumn 1997, with a small increment in January 1998 (Lavaniegos et al., 2002), while winter 2003 exhibited impoverished conditions, both in biomass and total abundance.

The seasonal changes in zooplankton biomass distribution showed the typical maximum in summer and minimum in winter (Lavaniegos et al., 1998), as well as the strong onshoreoffshore spring gradient. The anomalies in zooplankton las salinidades menores al promedio son el resultado de la ocurrencia de vientos hacia el ecuador más fuertes que lo normal, los cuales originaron un mayor transporte de agua de origen norteño. La ocurrencia de vientos con mayor intensidad es una condición típica presente durante eventos La Niña. Sin embargo, los datos de vientos mostrados en este trabajo y los diagramas T-S obtenidos durante 2002-2003 indicaron que las condiciones atmosféricas e hidrográficas fueron substancialmente diferentes a las observadas en La Niña 1999-2000 (Durazo et al., 2001; Durazo y Baumgartner, 2002). Durante La Niña, el incremento en los afloramientos eleva la nutriclina en la región costera de Baja California (Espinosa-Carreón et al., 2004) y transporta hacia la superficie agua de mayor salinidad, por lo que no se hace evidente el mínimo relativo de salinidad localizado entre 25 y $25.5 \sigma_{\mathrm{t}}$ que señala el núcleo de la CC (Durazo y Baumgartner, 2002).

La fuente del agua con baja salinidad en el Océano Pacífico nororiental es el agua del subártico (Lynn y Simpson, 1987), la cual ha estado influenciando al SCC desde el verano de 2002 (Huyer, 2003; Kosro, 2003; Venrick et al., 2003). Los resultados de este trabajo sugieren que el enriquecimiento de agua del subártico en las latitudes norteñas llegó tan al sur como los $28^{\circ} \mathrm{N}$ entre julio de 2002 y abril de 2003. Por lo tanto, la influencia de un El Niño débil podría haber sido enmascarada por la influencia del agua de origen subártico.

Por debajo de aproximadamente $100 \mathrm{~m}$ de profundidad el desarrollo de la CU y la dispersión de los datos en los diagramas T-S hacia salinidades mayores a lo largo de la $\sigma_{\mathrm{t}} 26-26.5$, sugirieron la presencia de un evento El Niño desde julio de 2002 hasta enero de 2003. La sincronización de las anomalías positivas observadas difirieron de lo encontrado por Venrick et al. (2003), quienes reportaron un El Niño moderado en las aguas del sur de California solamente para los primeros meses de 2003. Cuando se compararon con anomalías calculadas durante El Niño 1997-1998, las magnitudes de las anomalías de temperatura y salinidad $\left(1-2^{\circ} \mathrm{C}, 0.2-0.4\right)$ indicaron que el efecto durante 2002-2003 fue muy débil.

La información obtenida por medio de la clorofila $a$ apoyó también la ausencia de un calentamiento superficial asociado con condiciones El Niño. En abril de 2003 se presentó un incremento en la biomasa del fitoplancton en las estaciones costeras, dos veces mayor a lo observado por Lavaniegos et al. (2003) durante El Niño 1997-1998 para las aguas frente a Baja California. Este incremento de la concentración de clorofila en abril de 2003 podría ser interpretado como la terminación de El Niño, debido que desde enero/febrero de 2003 se tuvieron condiciones casi normales en las regiones oriental y central del Océano Pacífico ecuatorial. Para mayo de 2003 las condiciones de calentamiento anómalo habían desaparecido completamente (http://www.cpc.ncep.noaa.gov; McPhaden, 2004).

Los cambios en la biomasa del zooplancton siguieron un patrón similar, respondiendo al incremento en la clorofila con valores anómalamente altos en las estaciones costeras. Sin embargo, la situación fue claramente diferente a la observada en Baja California durante el fuerte evento El Niño 1997-1998 
distribution suggest that the transition from summer to autumn in 2002, as well as the transition from winter to spring in 2003, were more drastic than usual because of the presence of abundant salps in summer, decreasing thereafter. Salps are large pelagic tunicates, which impact the measurements based on biovolume. However, this acute transition from July to October 2002 does not appear to be influenced by a pattern typical of El Niño because of the unusual surface cooling observed during these months (e.g., Bograd and Lynn, 2003). A subarctic influence originated $10-\mathrm{m}$ temperature anomalies in the range of $-3^{\circ} \mathrm{C}$ to $0^{\circ} \mathrm{C}$ during cruise 0207 , and of $-2^{\circ} \mathrm{C}$ to $0^{\circ} \mathrm{C}$ during 0210. The species observed during El Niño 1997-1998 were related to saline water from the southwest (Hereu et al., 2003), while the blooms of Pegea socia present in northern Baja California during the summer of 2002 seem to be transported to the region from northern latitudes.

The zooplankton community structure of major taxa revealed drastic changes in tunicates and large carnivores, while other groups kept more conservative proportions. Tunicates are an important herbivorous assemblage in the CCS (Berner, 1967; Smith, 1985; Lavaniegos and Ohman, 2003), significantly altering the flux of matter in the pelagic ecosystem (Fortier et al., 1994). Although the causes triggering salps and other tunicate blooms must still be investigated, it has been suggested that these may be driven by periods of stratification followed by wind-induced mixing (Ménard et al., 1994). The thermal and saline fronts often recorded off Baja California (Roden, 1971) may provide the appropriate niches for the development of these organisms, which would find good conditions for grazing at moderate phytoplankton concentrations in the region. The ocean off Baja California is a very unstable area because of the strong replacement of species that occurs.

\section{Acknowledgements}

Funding was provided by CONACYT (projects G0041T, G35326-T, SEP-2003-C0242569 y U40822-F), CICESE and UABC (program 311). We are grateful to the captain and crew of the R/V Francisco de Ulloa for their help during the field work. Thanks to all the students and technicians that participated during the IMECOCAL surveys and the sample analyses. J. García processed all the CTD data. M. de la Cruz, J.M. Domínguez and F. Ponce worked on some figures. Wind data were obtained by E. Gil. Historical data of zooplankton volumes were provided by Paul Smith. The comments and suggestions made by three reviewers greatly improved this manuscript.

\section{References}

Berner, L.D. (1967). Distributional Atlas of Thaliacea in the California Current region. CalCOFI Atlas, 8: 1-322.

Bograd, S.J. and Lynn, R.J. (2003). Anomalous subarctic influence in the southern California Current during 2002. Geophys. Res. Lett., 30, 8020, doi: 10.1029/2003GL017446.
(Lavaniegos et al., 2002). La biomasa del zooplancton se mantuvo en sus niveles normales durante el otoño de 1997, con un pequeño incremento en enero de 1998 (Lavaniegos et al., 2002), mientras que en invierno de 2003 mostró condiciones de empobrecimiento, tanto en la biomasa como en la abundancia total.

Los cambios estacionales en la distribución de la biomasa del zooplancton mostraron los típicos máximos en el verano y mínimos en invierno (Lavaniegos et al., 1998), así como un fuerte gradiente costa océano en la primavera. Las anomalías en la distribución del zooplancton sugieren que la transición del verano al otoño en 2002, así como la transición del invierno a la primavera de 2003, fueron más drásticas que lo usual debido a la presencia de abundantes salpas en el verano, las cuales decrecieron posteriormente. Las salpas son grandes tunicados pelágicos que impactan las mediciones basadas en biovolumen. Esta transición aguda de julio a octubre no pareció ser influenciada por el patrón típico de El Niño dado al inusual enfriamiento superficial observado durante estos meses (ejemplo Bograd y Lynn, 2003). La influencia del agua subártica originó anomalías de la temperatura a $10 \mathrm{~m}$ en el intervalo de $-3^{\circ} \mathrm{C}$ a $0^{\circ} \mathrm{C}$ durante el crucero 0207 y de $-2^{\circ} \mathrm{C}$ a $0^{\circ} \mathrm{C}$ durante el crucero 0210. Las especies del zooplancton observadas durante El Niño 1997-1998 estuvieron asociadas al agua con mayor salinidad proveniente del suroeste (Hereu et al., 2003), mientras que los florecimientos de Pegea socia presentes en el norte de Baja California durante el verano de 2002 parecen ser transportados hacia la región desde latitudes norteñas.

Las estructura del zooplancton por grupos funcionales reveló cambios drásticos en tunicados y en carnívoros de gran tamaño, mientras que los otros grupos se mantuvieron en proporciones más conservadoras. Los tunicados son un grupo muy importante de herbívoros en el SCC (Berner, 1967; Smith, 1985; Lavaniegos y Ohman, 2003), los cuales alteran notablemente el flujo de la materia orgánica en el ecosistema pelágico (Fortier et al., 1994). No obstante, las causas por las que se incrementaron los florecimientos de salpas y otros tunicados todavía tienen que ser investigadas. Se ha sugerido que estos florecimientos podrían ser provocados por periodos de estratificación seguidos por la mezcla inducida por el viento (Ménard et al., 1994). Los frentes térmicos y salinos observados frecuentemente frente a Baja California (Roden, 1971) podrían proporcionar nichos adecuados para el desarrollo de estos organismos, los cuales encontrarían buenas condiciones para pastorear sobre concentraciones moderadas de fitoplancton en la región. El océano frente a Baja California es una zona de gran inestabilidad debido a que se presenta un fuerte reemplazo de las especies del zooplancton.

\section{Agradecimientos}

Este trabajo fue apoyado por el CONACYT (proyectos G0041T, G35326-T, SEP-2003-C0242569 y U40822-F), por el CICESE y la UABC (programa 311). Agradecemos al capitán y a la tripulación del B/O Francisco de Ulloa por su ayuda 
Bograd, S.J., DiGiacomo, P.M. Durazo, R., Hayward, T.L., Hyrenbach, K.D., Lynn, R.J., Mantyla, A.W., Schwing, F.B., Sydeman, W.J., Baumgartner, T., Lavaniegos, B. and Moore, C.S. (2000). The state of the California Current, 1999-2000: Forward to a new regime? CalCOFI Rep., 41: 26-52.

Durazo, R. and Baumgartner, T. (2002) Evolution of oceanographic conditions off Baja California: 1997-1999. Prog. Oceanogr., 54: 7-31.

Durazo, R., Baumgartner, T.R., Bograd, S.J., Collins, C.A., de la Campa, S., García, J., Gaxiola-Castro, G., Huyer, A., Hyrenbach, D., Loya, D., Lynn, R.J., Schwing, F.B., Smith, R.L., Sydeman, W.J. and Wheeler, P. (2001) The state of the California Current 2000-2001: A third straight La Niña year. CalCOFI Rep., 42: 29-60.

Espinosa-Carreón, T.L., Strub, T., Bier, E., Ocampo, F. and GaxiolaCastro, G. (2004). Seasonal and interannual variability of satellite derived chlorophyll, surface height and temperature off Baja California. J. Geophys. Res., 109, C03039, doi:10.1029/ 2003JC002105.

Fortier, L., Lefevre, J. and Legendre, L. (1994). Export of biogenic carbon to fish and to the deep-ocean. The role of large planktonic microphages. J. Plankton Res., 16: 809-839.

Hayward, T.L., Baumgartner, T.R., Checkley, D.M., Durazo, R., Gaxiola-Castro, G., Hyrenbach, K.D., Mantyla, A.W., Mullin, M.M., Murphree, T., Schwing, F.B., Smith, P.E. and Tegner, M.J. (1999). The state of the California Current in 1998-1999: Transition to cool-water conditions. CalCOFI Rep., 40: 29-62.

Hereu, C.M., Jiménez-Pérez, L.C. and Lavaniegos, B.E. (2003). Effects of the El Niño 1997-1998 and the rapid transition to cool conditions in the winter 1998-1999 on the copepods and salps of the California Current. 3rd International Zooplankton Production Symp., 20-23 May 2003, Gijón, Spain, Program and Abstracts, p. 53.

Holm Hansen, O., Lorenzen, C.J., Holmes, R.W. and Strickland, J.D.H. (1965). Fluorometric determination of chlorophyll. J. Cons. Int. Explor. Mer, 30: 3-15.

Huyer, A. (2003). Prefase to special section on enhanced subarctic influence in the California Current, 2002. Geophys. Res. Lett., 30, 8019, doi:10.1029/2003GL017724.

Kosro, P.M. (2003). Enhanced southward flow over the Oregon shelf in 2002: A conduit for subarctic water. Geophys. Res. Lett., 30, doi: 10.1029/2003GL017436.

Lagerloef, G.S.E., Lukas, R., Bonjean, F., Gunn, J.T., Mitchum, G.T., Bourassa, M. and Busalacchi, J. (2003) El Niño tropical Pacific Ocean surface current and temperature evolution in 2002 and outlook for early 2003. Geophys. Res. Lett., 30, 1514, doi:10.1029/2003GL017096.

Lavaniegos, B.E. and Ohman, M.D. (2003). Long-term changes in pelagic tunicates in the California Current. Deep-Sea Res. II, 50: 2493-2518.

Lavaniegos, B.E., Gómez-Gutiérrez, J., Lara-Lara, J.R. and Hernández-Vázquez, S. (1998). Long-term changes of zooplankton volumes in the California Current System. The Baja California region. Mar. Ecol. Prog. Ser., 169: 55-64.

Lavaniegos, B.E., Jiménez-Pérez, L.C. and Gaxiola-Castro, G. (2002). Plankton response to El Niño 1997-1998 and La Niña 1999 in the southern region of the California Current. Prog. Oceanogr., 54: 33-58.

Lavaniegos, B.E., Gaxiola-Castro, G., Jiménez-Pérez, L.C., GonzálezEsparza, M.R., Baumgartner, T. and J. García-Córdova (2003). 1997-98 El Niño effects on the pelagic ecosystem of the California Current off Baja California, Mexico. Geofís. Int., 42: 483-494. durante los cruceros. Damos las gracias a todos los estudiantes y técnicos participantes en el IMECOCAL por su ayuda durante los muestreos y en el análisis de muestras. J. García procesó todos los datos del CTD; M. de la Cruz, J.M. Domínguez y F. Ponce ayudaron con algunas figuras. Los datos de viento fueron obtenidos por E. Gil. Los datos históricos del volumen del zooplancton fueron proporcionados por P. Smith. Los comentarios y sugerencias de tres revisores ayudaron a mejorar este trabajo.

Traducido al español por los autores.

Lynn, R.J. and Simpson, J.J. (1987) The California Current system: The seasonal variability of physical characteristics. J Geophys. Res., 92: 12947-12966.

Lynn, R.J. and Bograd, S. (2002). Dynamic evolution of the 19971999 El Niño-La Niña cycle in the southern California Current System. Prog. Oceanogr., 54: 59-75.

Lynn, R.J., Baumgartner, T., García, J., Collins, C.A., Hayward, T.L., Hyrenbach, K.D., Mantyla, A.W., Murphree, T., Shankle, A., Schwing, F.B., Sakuma, K.M. and Tegner, M.J. (1998). The state of the California Current, 1997-1998: Transition to El Niño conditions. CalCOFI Rep., 39: 25-49.

McPhaden, M.J. (2004). Evolution of the 2002-2003 El Niño. Bull. Am. Meteor. Soc., 85, 677, doi:10.1175/BAMS-85-5-677.

Ménard, F., Dallot, S., Thomas, G. and Braconnot, J.C. (1994). Temporal fluctuations of two Mediterranean salp populations from 1967 to 1990. Analysis of the influence of environmental variables using a Markov chain model. Mar. Ecol. Prog. Ser., 104: 139-152.

Palacios-Hernández, E., Argote, M.L., Amador, A. y Mancilla, M. (1996). Simulación barotrópica inducida por viento en Bahía Sebastián Vizcaíno, B.C. Atmósfera, 9: 171-188.

Roden, G.I. (1971). Aspects of the transition zone in the northeastern Pacific. J. Geohys. Res., 76: 3462-3475.

Schwing, F.B., Murphree, T., de Witt, L. and Green, P.M. (2002). The evolution of oceanic and atmospheric anomalies in the northeast Pacific during the El Niño and La Niña events of 1995-2001. Prog. Oceanogr., 54: 459-491.

Smith, P.E. (1985). A case history of an anti-El Niño to El Niño transition on plankton and nekton distribution and abundances. In: W.S. Wooster and D.L. Fluharty (eds.), El Niño north - Niño effects on the eastern subarctic Pacific Ocean. Washington Sea Grant Program, Seattle, pp. 121-142.

Smith, P.E. and Richardson, S.L. (1977). Standard techniques for pelagic fish egg and larva surveys. FAO Fish. Tech. Pap., 175: 1100.

Strub, P.T. and James, C. (2003). Altimeter estimates of anomalous transports into the California Current during 2000-2002. Geophys. Res. Lett., doi: 10,1029/2003GL017513.

Venrick, E.L. and Hayward, T.L. (1984). Determining chlorophyll on the 1984 CalCOFI surveys. CalCOFI Rep., 25: 74-79.

Venrick, E., Bograd, S., Checkley, D., Cummings, S., Durazo, R., Gaxiola-Castro, G., Hunter, J., Huyer, A., Hyrenbach, K.D., Lavaniegos, B.E., Mantyla, A., Schwing, F.B., Smith, R.L., Sydeman, W.J. and Wheeler, P.A. (2003). The state of the California Current, 2002-2003: Tropical and subarctic influences vie for dominance. CalCOFI Rep., 44: 28-60.

Yentsch, C.S. and Menzel, D.W. (1963). A method for the determination of phytoplankton chlorophyll and phaeophytin by flourescence. Deep-Sea Res., 10: 221-231. 